\title{
Performance of signal processing techniques for anomaly detection using a temperature-based measurement interpretation approach
}

\author{
Rolands Kromanis ${ }^{1}$ (D) Prakash Kripakaran ${ }^{2}$
}

Received: 2 May 2020 / Revised: 8 August 2020 / Accepted: 28 August 2020 / Published online: 10 September 2020

(c) The Author(s) 2020

\begin{abstract}
This study investigates the effectiveness of four signal processing techniques in supporting a data-driven strategy for anomaly detection that relies on correlations between measurements of bridge response and temperature distributions. The strategy builds upon the regression-based thermal response prediction methodology which was developed by the authors to accurately predict thermal response from distributed temperature measurements. The four techniques that are investigated as part of the strategy are moving fast Fourier transform, moving principal component analysis, signal subtraction method and cointegration method. The techniques are compared on measurement time histories from a laboratory structure and a footbridge at the National Physical Laboratory. Results demonstrate that anomaly events can be detected successfully depending on the magnitude and duration of the event and the choice of an appropriate anomaly detection technique.
\end{abstract}

Keywords Structural health monitoring $(\mathrm{SHM}) \cdot$ Signal analysis $\cdot$ Signal processing $\cdot$ Damage detection $\cdot$ Long term monitoring - Thermal effects

\section{Introduction}

Effective data interpretation approaches $[1,2]$ are key to support decision-making based on long-term bridge monitoring systems [3-5]. Such systems typically collect dynamic [6, 7] or quasi-static response measurements [8, 9], alongside environmental data such as temperature, humidity and wind. The collected response measurements, while including the effects of live loads and weather-related loads such as wind, are mostly dominated by the effects of daily and seasonal variations in ambient temperature [7-11]. Bridges have also been observed to have non-linear temperature gradients that often cause thermal stresses comparable to those due to live loads [12]. Therefore, reliable techniques for interpreting measurements must include appropriate ways of incorporating temperature effects.

The techniques used for measurement interpretation are usually referred to as structural identification

Rolands Kromanis

r.kromanis@utwente.nl

1 Faculty of Engineering Technology, University of Twente, Enschede, The Netherlands

2 College of Engineering, Mathematics and Physical Sciences, University of Exeter, Exeter, UK
(St-Id) techniques due to their use of system identification approaches [13, 14]. St-Id aims to develop numerical models that are capable of accurately predicting structural behaviour using measurements from structural health monitoring (SHM) [13]. Historically, in the context of aerospace and mechanical systems, St-Id techniques have been applied primarily for damage identification. Conceptually, damage identification can be considered to be part of a broad measurement interpretation paradigm that has the following five steps, where the first four are part of St-Id [15] and the last step is for the residual life prediction of a structure [16]:

- Detection. Detect anomalous behaviour (damage) of a structure.

- Localisation. Indicate the location of the damage.

- Classification. Determine the type of damage.

- Assessment. Assess the extent and severity of the damage.

- Prediction. Determine the fitness of the bridge and give a prognosis of its residual life.

In this paper, the focus is on the first two steps: detection and localisation of damage.

St-Id techniques can be broadly classified into two categories: (1) data driven and (2) model based [17]. The former 
class of techniques requires minimal structural knowledge and is better suited for continuous monitoring applications since they avoid behaviour models (e.g. finite element models), which are often difficult to generate and require significant computational resources. Data-driven methods generally employ statistical techniques to analyse the continuously collected measurements [18-21]. The goal is often to detect sudden or gradual changes in structural behaviour from shifts and drifts in measurement (or signal) patterns. However, these methods may not fully explain the reason for the observed change in behaviour.

Data-driven methods rely on the statistical relationships between distributed measurements. Posenato et al. [22] compared several signal processing techniques on a set of strain time histories and showed that moving principal component analysis (MPCA) offers the most promise for anomaly detection, which indicates a change in the performance of the structure or damage. However, MPCA failed to detect changes in signals unless damage is either close to the sensor location or is of very high severity [23]. A key reason cited for the lack of sensitivity of MPCA was the effects of ambient temperature variations on structural response. This was verified by [24] who showed on a laboratory structure that anomaly detectability improves significantly when thermal effects have been purged from measurements. They also put forward a temperature-based measurement interpretation (TB-MI) approach [25] a coupled data-driven anomaly detection strategy that adopts the principles of temperaturebased SHM [26]. The first step in this approach is to predict thermal response of a bridge from distributed temperature measurements. Then, prediction error signals, which are computed from the difference between predicted and measured response, are analysed for anomalies using anomaly detection techniques.

While the basic concept in the TB-MI approach was illustrated in [25], there has not been an in-depth investigation into the performance of various signal processing methods within this concept. This is the specific novelty of this paper, which focuses on the performance of four anomaly detection techniques within the TB-MI approach: (1) cointegration, (2) signal subtraction method, (3) MPCA and (4) moving fast Fourier transform are chosen. These techniques have been chosen for the superior performance they have demonstrated in previous studies [22, 23, 25, 27] on anomaly detection from long-term measurements. The capability of these techniques to detect sudden and gradual changes in structural performance (i.e. damage) is evaluated and compared using measurements from a laboratory truss and a concrete footbridge. The performance of the TB-MI approach is also compared with direct application of anomaly detection techniques on response measurements.

\section{Data-driven measurement interpretation approach}

The TB-MI approach consists of two parts. The first part is the regression-based thermal response prediction (RBTRP) methodology. This methodology utilises distributed temperature and response measurements to generate regression models that can predict thermal response from distributed temperature measurements [28]. The latter part analyses the prediction errors for anomaly events and locations.

\subsection{Regression-based thermal response prediction (RBTRP) methodology}

The RBTRP methodology aims to produce reliable regression models which predict bridge response from distributed temperature measurements. The methodology has a model generation phase and a model application phase. In the model generation phase, models for accurately predicting thermal response are generated. The main steps in this phase are:

1. Selection of a reference set of measurements. The selected (input) measurements are used to train regression models.

2. Data pre-processing. Measurements are cleansed from outliers using inter-quartile range technique, smoothed with a moving average filter and down-sampled to ensure input data quality for model training.

3. Dimensionality reduction of the measurement dataset using principal component analysis (PCA). Temperature measurements are transformed to the principal component space, and only those principal components that are sufficient to explain $99.9 \%$ of the variance in the temperature measurements are chosen as input to the regression models.

4. Generation and evaluation of statistical models for identifying the most accurate and robust models.

In the model application phase, statistical models identified in the model generation phase are employed to predict real-time response from distributed temperature measurements. Readers seeking more information on the RBTRP methodology are advised to refer to [28].

\subsection{Anomaly detection}

Traditional anomaly detection techniques analyse patterns derived from new measurements against patterns observed during a reference period for deviations from normal (baseline) behaviour. However, in this research, prediction error (PE) signals which are generated by the 
RBTRP methodology are analysed using signal processing techniques to detect anomalies which correspond to anomalous structural behaviour or structural damage. The reference period for anomaly detection is kept the same as the period used for training regression models in the RBTRP methodology. Patterns in the PE signals in the reference period are assumed to represent the baseline conditions of the structure. An anomaly is said to be detected when the deviations in measurement patterns, which are evaluated in relation to patterns present during the reference period, exceed the confidence interval that is determined based on the characteristics of the baseline patterns.

A schematic diagram of the anomaly detection process is shown in Fig. 1. Similar to the RBTRP methodology, there are two phases in the anomaly detection technique. Initially, baseline conditions of a structure are identified in the form of patterns derived from the PE signals corresponding to the reference period. This phase is represented as baseline condition identification. Subsequently, PEs computed from newly collected measurements and predicted response are examined for anomaly events. This phase is denoted as the real-time anomaly detection.

The identification of baseline conditions phase includes the following steps:

1. Pre-processing of PE signals. PE signals are pre-processed for outliers and noise. Outliers are removed using inter-quartile range technique. Signal noise is reduced using moving average filters.

2. Selection of an anomaly detection technique. A suite of signal processing techniques is employed to interpret PE signals. The techniques can be classified under the following two categories:

(a) Univariate signal analysis. Signal processing techniques such as moving fast Fourier transform (MFFT) [23] analyse the PE signals for each sensor individually. These techniques are useful to detect faulty sensors or damage that is very local to a sensor.

(b) Multivariate signal analysis. These techniques enable integrated analysis of time series of several parameters. These are useful for data interpretation in large, complex structures that have vast numbers of sensors. In such structures, clustering sensors into groups according to their correlations or other metrics, and then analysing these clusters for changes in correlations can reveal anomalies and indicate their locations [29, 30].

3. Selection of a training set. A training set is specified for the chosen anomaly detection technique. The PE values in the training set are used to derive key features as described in the next step.

4. Generation of statistical features. All signal processing techniques used for anomaly detection in this research rely fundamentally on statistical features, which define the patterns in the PE signals. In this step, features that are tracked by the chosen signal processing technique are evaluated from the values for the PE signal in the training set.

5. Determination of the confidence interval. This step involves evaluating the confidence interval or threshold bounds for the statistical features identified in the previous step. The thresholds are determined probabilistically based on the values for the remaining statistical features in the reference set.

The real-time anomaly detection phase denotes the application of signal processing techniques for real-time anomaly detection. It includes the following steps:

1. Pre-processing of PE values. PE values are pre-processed as in the identification of baseline conditions phase.

2. Computation of statistical features. This step involves computing values for statistical features used in the selected anomaly detection technique from $\mathrm{PE}$ values evaluated for newly collected measurements.

3. Classification of new measurements. This step evaluates if patterns derived from PEs are within the establish confidence interval, and based on this evaluation, classifies new measurements as representative of either anomalous or normal structural behaviour.

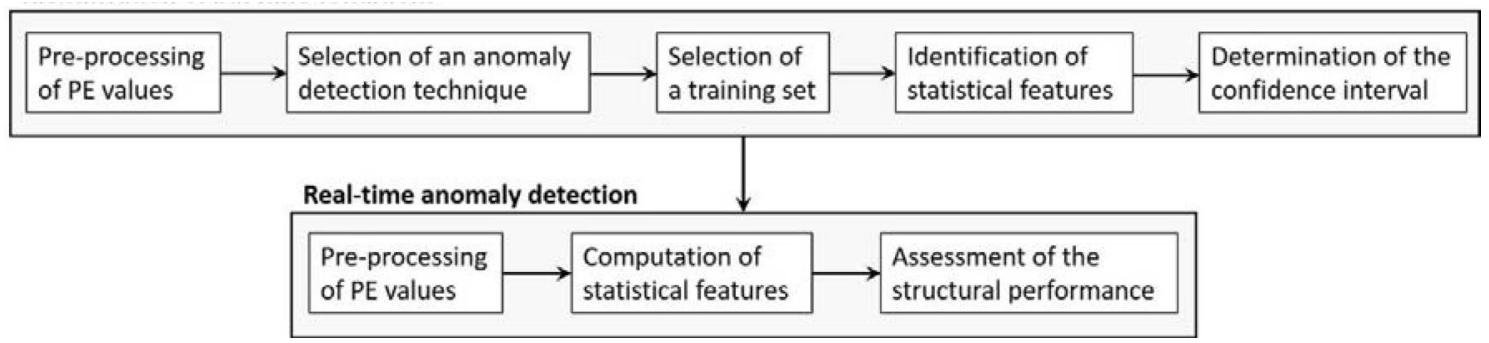

Fig. 1 Flowchart of anomaly detection process 


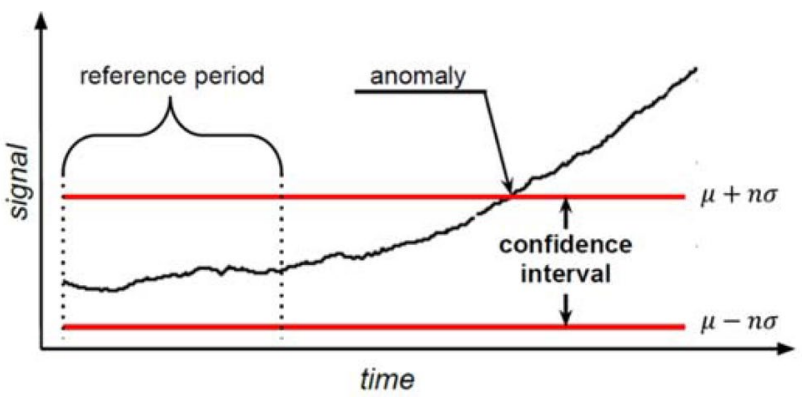

Fig. 2 Determination of baseline conditions

Figure 2 graphically illustrates the classification step, which is described above as the last step in the real-time anomaly detection phase, on an arbitrary PE signal. An anomaly is likely to indicate damage when the feature tracked by the anomaly detection technique departs irreversibly outside of the confidence interval. The confidence interval for the statistical features can be defined assuming the parameters of the features are Gaussian variables. For example, they can be specified as $[\mu-n \sigma, \mu+n \sigma]$, where $\mu$ and $\sigma$ are the mean and standard deviation of the values for the signal derived using the selected technique during the reference period, and $n$ is an integer value greater than zero. Defining the confidence interval closer to the mean value of the signal, i.e. by choosing a small value for $n$, increases the likelihood of false positives and false negatives. In contrast, larger threshold bounds, i.e. by choosing large values for $n$, imply that only anomaly events of high severity are detected [27]. Commonly used values for $n$ are 3 and 6, which correspond to confidence levels of $99.73 \%$ and $99.99 \%$ for signals representing a Gaussian process $[22,30,31]$. Therefore, the specification of threshold bounds, and by implication, selection of the training set has to be done prudently based on the statistical data and the nature of the signal.

The classification step, as illustrated in Fig. 2 and discussed above, is structured in a simplistic manner, although, in reality, it can be more complex and complicated. This step can be related to visualising results for bridge operators, and therefore, a careful consideration has to be given to how the results from anomaly detection are presented. For example, the classification step can be probabilistic in nature and suggest the likelihood that new measurements are representative of anomalous structural behaviour. However, this research focuses only on the application of anomaly detection techniques; human-computer interaction and results visualisation are considered outside the scope of this work.

\subsection{Signal processing techniques}

In the following sections, four signal processing techniques, which are grouped as univariate and multivariate signal analysis techniques in this research, are described. Two of the techniques-moving fast Fourier transform and signal subtraction method, have been introduced by the authors and have shown promise for analysing SHM data [23, 25]. The other two techniques-moving principal component analysis and cointegration, have been chosen out of several investigated in previous research due to the superior performance they have demonstrated for damage detection from longterm quasi-static response time histories [22, 27].

\subsubsection{Univariate signal analysis}

These signal processing techniques analyse each PE signal individually, and are, hence, appropriate for detecting faulty sensors or anomalies in univariate signals. The interpretation of a single signal also requires less computational effort than integrated analysis of multiple PE signals.

Moving fast Fourier transform (MFFT). Fourier transforms are generally used to transform signals from time domain to frequency domain by determining their frequency content, and also the relative magnitudes of the various frequencies [32]. MFFT is the fast Fourier transform of a moving window of data points from a time series, which in the case of this research is the PE signal. When applying MFFT for anomaly detection, the frequency content of the PE signal is tracked to identify changes in structural performance. Specifically, the amplitude of the lowest frequency component is considered [23]. An example of the application of MFFT is illustrated conceptually in Fig. 3 using an arbitrary PE signal.

\subsubsection{Multivariate signal analysis}

The measured response at a particular location of a bridge is often correlated with other response measurements taken in its vicinity [33]. Multivariate signal processing techniques take advantage of spatial correlations in signals. They can be superior to univariate signal analysis techniques when evaluating strongly correlated signals such as from SHM. Multivariate signal analysis techniques are capable of resolving such environmental and operational variations by using spatial correlations. This research employs three multivariate signal processing techniques: (1) signal subtraction method, (2) moving principal component analysis and (3) cointegration. 
Fig. 3 Application of the MFFT on a PE signal

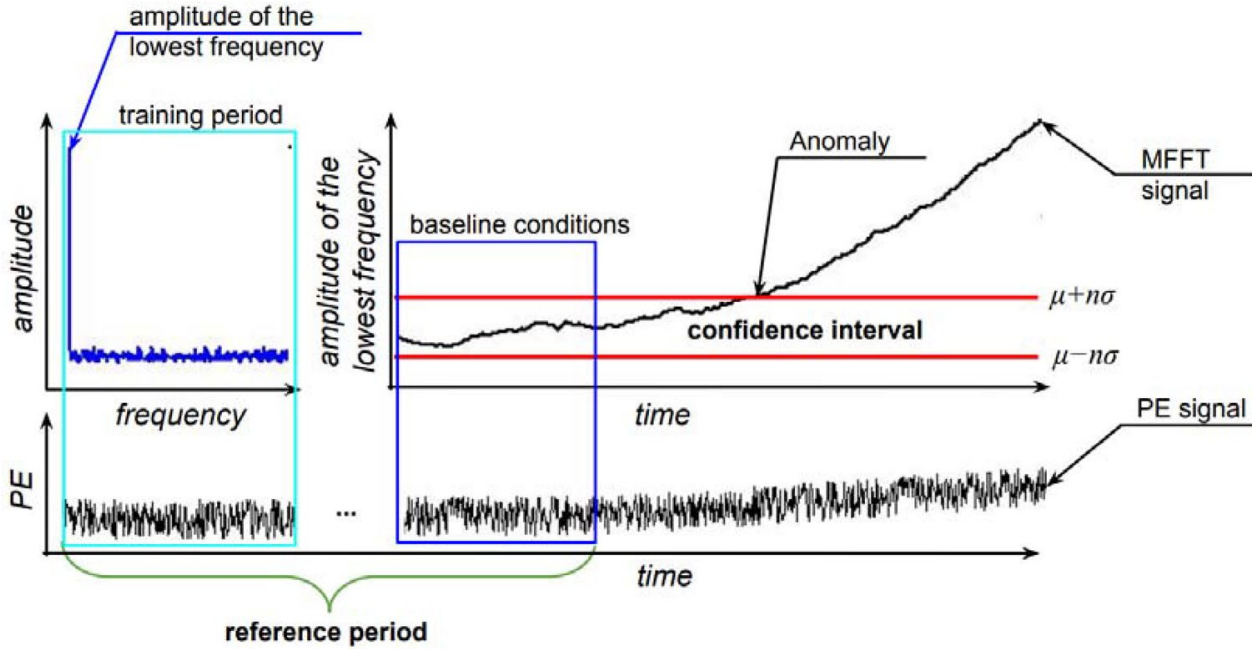

Signal subtraction method (SSM). SSM is a technique proposed in [25], in which two PE signals are linearly combined to generate a new signal, which is then analysed for anomalies. Mathematically, it is applied as follows:

$T_{k l}=\left(\frac{w_{k}}{r_{k}}\right) \Delta y_{k}-\left(\frac{w_{l}}{r_{l}}\right) \Delta y_{l}$,

where $T_{k l}$ is the new signal resulting from the subtraction process. $\Delta y_{k}$ and $\Delta y_{l}$ are values of the PE signals corresponding to sensors $k$ and $l$, respectively. $r_{k}$ and $r_{l}$ are scaling factors for the two PE signals. These are equal to the range of signal values in the training period, i.e. the difference between the maximum and minimum values in the training period. $w_{k}$ and $w_{l}$ are weights specified according to the accuracies of the respective sensor and its corresponding model for thermal response prediction. In this study, the hypothesis is that measurements from all elements are equally important. Therefore, weights of all PE signals are set equal to 1 .

Moving principal component analysis (MPCA). MPCA was originally proposed for anomaly detection by Posenato et al. [34]. Its anomaly detection capabilities were shown to be superior in comparison to a number of other techniques such as ARIMA and wavelets [22]. MPCA is fundamentally an extension of PCA, which is a statistical technique to reduce the dimensionality of large datasets [35]. PCA involves finding a set of ordered orthonormal vectors referred to as principal components such that a few vectors explain nearly all the variability in the datasets. Application of MPCA to a cluster of time series essentially involves the iterative application of PCA over arrays of data obtained from windows moving incrementally over a cluster of time series. The moving window concept is the same as for MFFT (see Fig. 3); however, MPCA is applied simultaneously on multiple signals.
In this study, MPCA is applied to a cluster of PE signals. Changes to the principal components indicate changes in the correlations between the PE signals and, hence, can imply the onset of anomalous structural behaviour or damage. For structures that are monitored with a vast number of sensors, the process of clustering PE signals is a crucial step, and can affect significantly the performance of anomaly detection. Posenato et al. [34] in their investigation on using MPCA to analyse response measurements suggested a simple heuristic that uses the correlations between measurements from various sensors to arrive at the number and composition of clusters. The idea is to cluster measurement time series that are strongly correlated, and the corresponding sensors can usually be identified using engineering judgement.

Cointegration. The cointegration technique utilises the statistical properties of cointegrated signals for anomaly detection. Measurement time series of bridge response (signals) follows daily and seasonal temperature trends. Such time series can be classified as non-stationary processes. A nonstationary signal is said to be integrated to an order $d$, if a process of taking differences over the time series repeated $d$ times leads to a stationary signal. In mathematical notation, the order of integration of a signal is often denoted by $I(d)$. When considering a cluster of signals, the cluster is said to be cointegrated if there exists a linear combination of the component variables (measurements) that are stationary. The resulting stationary signals are referred to as cointegrated signals. This technique, initially proposed and used in the field of econometrics [36], has been introduced for SHM by Cross et al. [27]. It has been shown to be useful for purging quasi-static effects in measurements, and has been demonstrated on a few benchmark problems, and on measurements from the NPL Footbridge [37]. Applications of cointegration for damage detection in long-term measurements have also been expanded to nonlinear approaches [38], multiresolution 
approaches [39] and regime-switching methods [30]. Mathematics behind cointegration is not detailed in this paper. Interested readers are referred to [27].

\section{Case studies}

A laboratory truss and the National Physical Laboratory (NPL) Footbridge are selected to assess the anomaly detection capabilities of the proposed measurement interpretation approach.

\subsection{Laboratory truss}

A 3-m-long aluminium laboratory truss, which was manufactured at the University of Exeter, UK, and has served as a test-bed for studies by Kromanis and Kripakaran [24, 25], is considered in this study (see Fig. 4). The truss is built of aluminium sections. Top and bottom chords and outer diagonals are composed of two channel section forming an "I" section. Other elements are flat bars. All elements are joined with six bolts. The truss is connected to the concrete foundation with both supports being pinned. Three thermal heaters are installed $0.5 \mathrm{~m}$ above and $0.3 \mathrm{~m}$ away from the truss. Heaters are periodically turned on and off to create additional temperature loads to already existing ambient temperature.

The truss is monitored with 31 thermocouples and 10 strain gauges (see Fig. 5). Measurements are collected at 10 -s intervals for a period of 13 days. Continuous time histories of temperatures and strains are plotted in Fig. 6. Damage is created by removing bolts and releasing the right support of the truss. All events are listed in Table 1.

\subsection{NPL footbridge}

The NPL footbridge was located on the premises of the National Physical Laboratory in Teddington, UK. The footbridge, which had been in service for more than 40 years, was moved from its original location in 2008 and setup as a SHM demonstrator (see Fig. 7). The deck of the bridge is 20-m long and it is supported on two "A" shaped columns

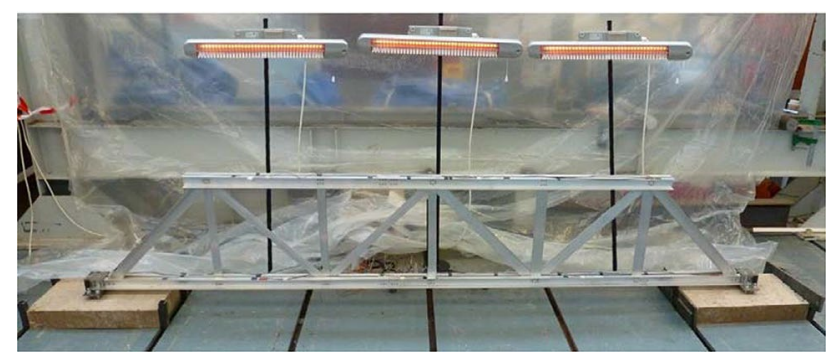

Fig. 4 A photograph of the truss

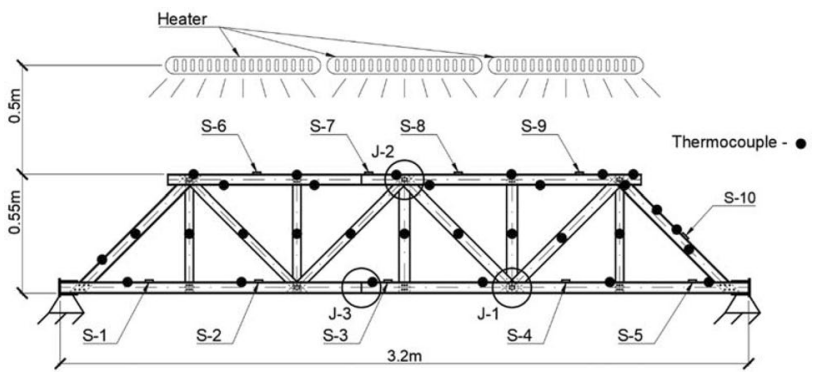

Fig. 5 A sketch of the truss showing its principal dimensions, boundary conditions, location of sensors ( $S$ - strain gauge, $i=1,2, \ldots, 10)$, damaged joints ( $J$-joint, $i=1,2,3)$ and heaters

standing $10.5 \mathrm{~m}$ apart (see Fig. 8). The footbridge is equipped with a wide range of sensing units [40]. A series of experimental activities including load tests and strengthening procedures have been carried out on the footbridge on a relatively regular basis. The activities and their times, which are pertinent to the duration of data used in this study, are listed in Table 2. The table also provides the notation that are used to refer to the various events when analysing the results.

In this study, tilt measurements from eight electrolevel tilt sensors and temperatures from the thermistors in ten vibrating-wire arc-weldable strain gauges are used to demonstrate the measurement interpretation approach, specifically, anomaly detection. The tilt sensors have resolution of $5.2 \times 10^{-3} \mathrm{~mm} / \mathrm{m}$. The resolution of the thermistors is $\pm 0.01{ }^{\circ} \mathrm{C}$. The locations of these sensors are indicated in Fig. 8. Since strain gauges are placed at the bottom of the handrails and not directly on the deck, measured temperatures are unlikely to represent the temperatures at the locations where the tilt measurements are collected particularly given the distinct thermal conductivity characteristics of the materials of the metal handrail and the concrete deck. Therefore, predicting the response of this footbridge from the measured temperatures is a challenging task.

\subsubsection{Measurement time histories}

Data collected from nearly two and a half years of monitoring of the footbridge are selected. Over the course of monitoring, measurements have generally been collected at a rate of one measurement per minute but the rate has also occasionally been reduced to one measurement per hour. There are also periods when data collection was interrupted for several days. Maintaining a consistent measurement frequency is, however, crucial to identifying patterns in the data. Computational requirements can also be reduced by eliminating excess data. Portions of the original dataset where one measurement is taken every minute is, therefore, down-sampled to create a new dataset resembling a measurement frequency of one measurement 

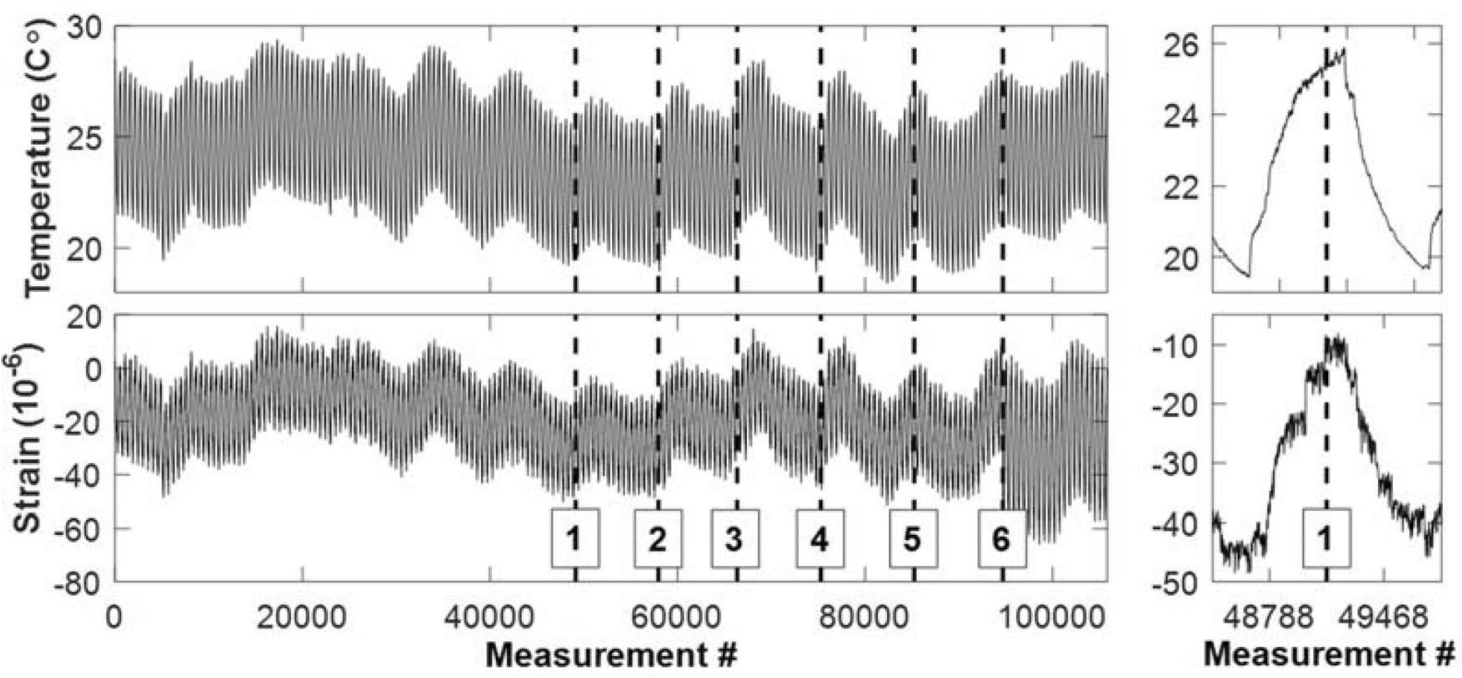

Fig. 6 Time-histories of temperatures at the bottom chord (top) and strains (bottom) measured with S-3 with zoomed-in views for a simulated daily cycle (right) around the time of damage event \#1. Numbers in the boxes correspond to events listed in Table 1

Table 1 The lists of events for the truss

\begin{tabular}{|c|c|c|c|c|c|c|}
\hline Event & 1 & 2 & 3 & 4 & 5 & 6 \\
\hline Affected joints & $\mathrm{J}-1$ & $\mathrm{~J}-1$ & $\mathrm{~J}-1, \mathrm{~J}-2$ & $\mathrm{~J}-1, \mathrm{~J}-2, \mathrm{~J}-3$ & $\mathrm{a}$ & b \\
\hline $\begin{array}{l}\text { Number (\#) of removed } \\
\text { bolts }\end{array}$ & 3 & 5 & 8 & 11 & 0 & 0 \\
\hline
\end{tabular}

${ }^{\mathrm{a}}$ All connections are repaired, i.e. bolts are put back

${ }^{\mathrm{b}}$ Right support is released

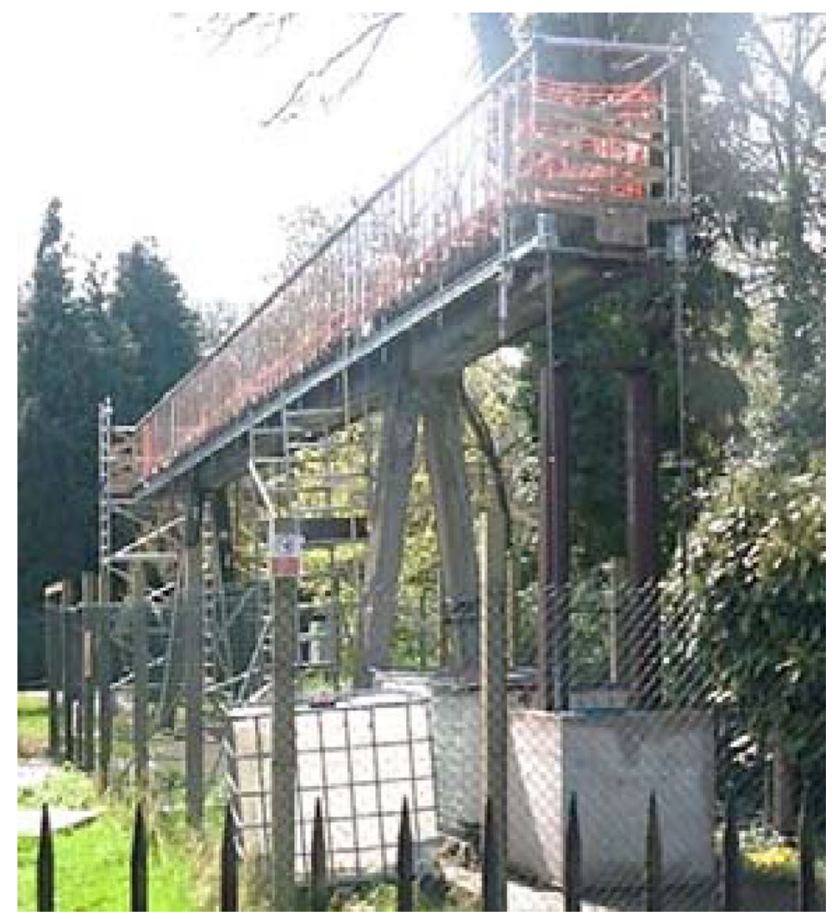

every 5 min. Durations corresponding to no measurement periods are removed. The periods when measurements were taken at a rate of one measurement every hour comprise a small part of the entire measurement set $(<2 \%)$ and are not up-sampled further. This procedure has been shown to be appropriate for generating regression models for thermal response prediction from the NPL footbridge data [28].

Time-histories of temperatures and strains are pre-processed. Temperatures measured with sensor TEMP-1 are plotted in Fig. 9. These show a typical sinusoidal trend indicating seasonal temperature variations, but when examined closer (see Fig. 9 (right)) also have smaller sinusoidal cycles from daily temperature variations. Tilts measured by sensors TL-5, TL-6, TL-7 and TL-8 are plotted in Fig. 10. Plots of measurements from sensors TL- 5 and TL- 6 show that tilts of the deck, while being correlated with temperatures during the summer times, do not strictly follow temperature variations during the winter periods. Instead, the magnitude of the tilts shows little variation during winters (from October to March). This phenomenon is possibly due to

Fig. 7 A photograph of the NPL footbridge 
Fig. 8 A sketch of the NPL footbridge. TL-i $(i=1,2, \ldots$, 8) and TEMP-j $(j=1,2, \ldots$, 10) indicate the locations of tilt sensors and thermistors, respectively

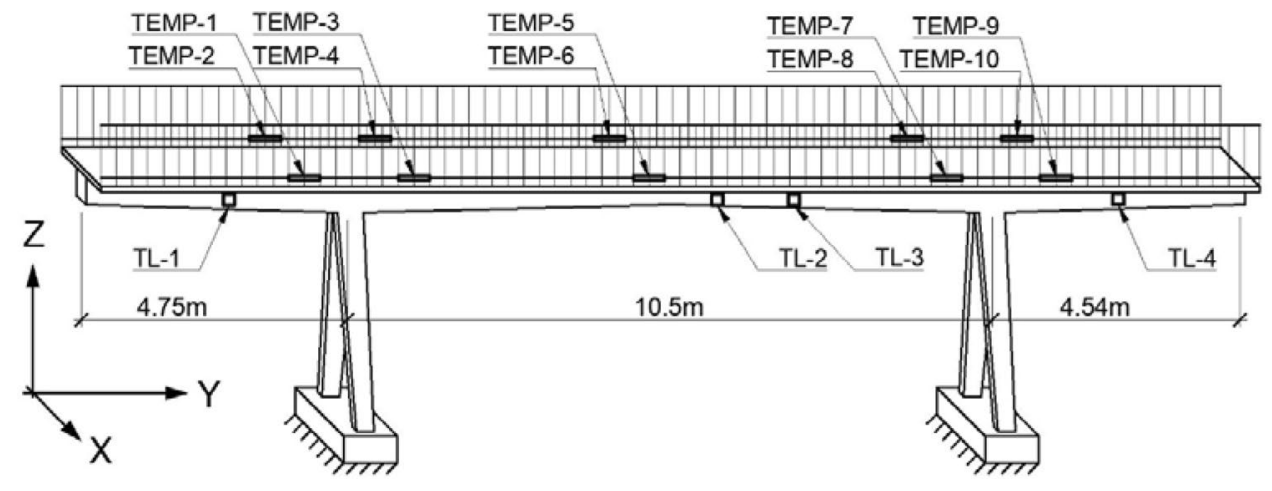

Table 2 The list of events for the NPL footbridge

\begin{tabular}{|c|c|c|}
\hline Event \# & Date & Activities \\
\hline 1 & March 24, 2009 & \multirow{3}{*}{$\begin{array}{l}\text { Static tests-filling and emptying } \\
\text { water tanks which are attached to } \\
\text { the left cantilever }\end{array}$} \\
\hline 2 & June 29-30, 2009 & \\
\hline 3 & August 1-3, 2009 & \\
\hline 4 & June 30-July 2, 2010 & Static tests and scaffolding activities \\
\hline 5 & October 18, 2010 & $\begin{array}{l}\text { Cut in the left cantilever and static } \\
\text { tests }\end{array}$ \\
\hline 6 & November 1, 2010 & Cantilever repaired \\
\hline 7 & April 28, 2011 & Cut in the left cantilever \\
\hline 8 & June 27, 2011 & Rebar cut \\
\hline
\end{tabular}

- the limited solar radiation during the winter periods resulting in small thermal gradients across the deck cross-section and therefore causing minimal longitudinal bending of the structure

- or the fact that the bottom part of the "T"-shaped deck is an arch and it does not bend inwards; hence, measured tilts are minimal.

Sensors TL-7 and TL-8 measure tilts of the "A" shaped columns about the y-axis. Measurements from TL-7 show sinusoidal trends resembling both daily and seasonal temperature variations. On the other hand, measurements from
TL-8, while having some sinusoidal trends, increase in magnitude over the course of the monitoring. This may be due to either a sensor malfunction or from ongoing settlement of the foundation supporting this column.

\subsubsection{Event histories}

During the monitoring, the footbridge was exposed to a variety of loading and cut-and-repair tests. Details of such events that may have changed the performance of the footbridge are listed in Table 2. While temperature variations explain patterns in tilt measurements (with some exceptions, such as from TL-8), a closer examination, particularly with knowledge of the activities on the bridge, can help to identify the events. For example, measurements collected with TL-5 are shown up close in Fig. 11. The effects of events \#2 and \#3 can be identified from Fig. 10 by looking at measurements at the corresponding timeframe. The two drops in tilt measurements due to these events are indicated by circles in Fig. 11. Both events \#2 and \#3 are static load tests. In these tests (see Table 2), a load was applied at the end of the left cantilever portion of the footbridge. Identifying such events from measurement time histories is a difficult task, especially without having a priori knowledge of times and nature of the activities.
Fig. 9 Temperatures measured by TEMP- 1 over the selected monitoring period (left) and one day (right)
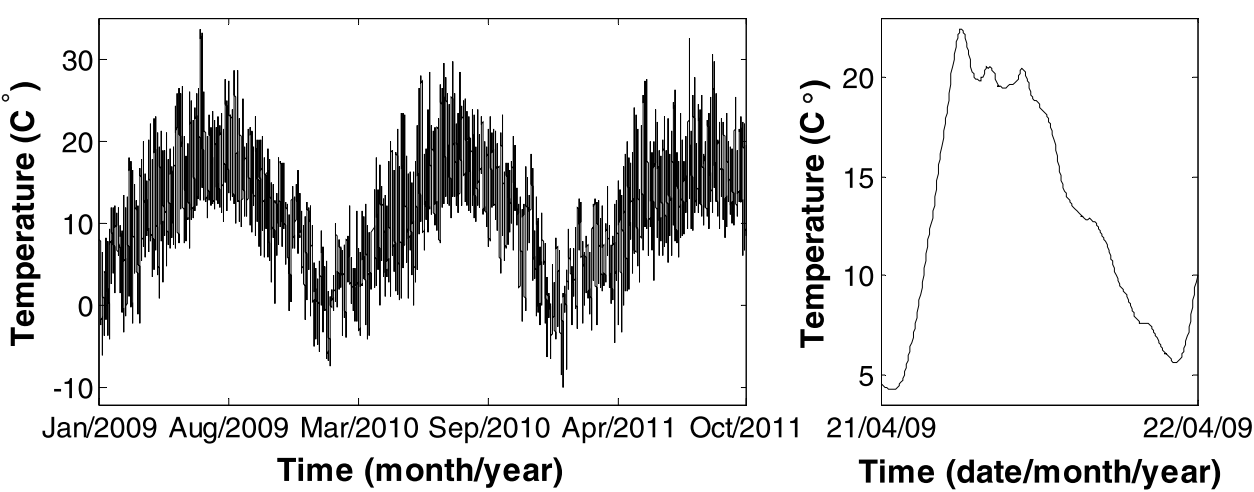
Fig. 10 Time histories of selected tilt measurements
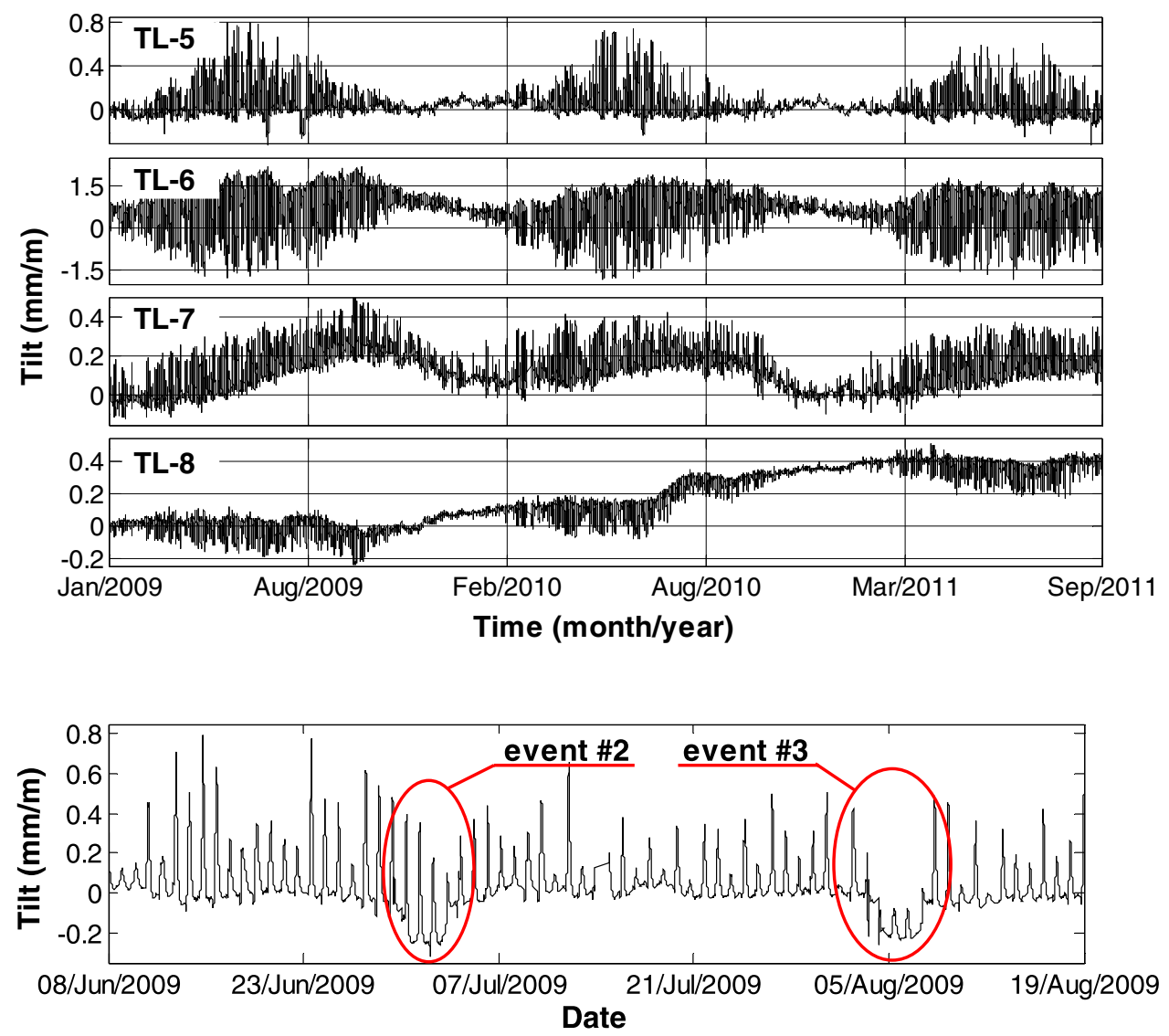

Fig. 11 Time histories of TL-5 between 08/Jun/2009 and 19/ Aug/2009. Periods referring to events \#2 and \#3 are circled dataset is selected to generate and evaluate regression models under the RBTRP methodology. Selected PE signals (from sensor locations close to the damage) are shown in Fig. 12. PE signals related to a particular sensor location are named as PE \{sensor name\}. When visually inspecting $\mathrm{PE}$ signals corresponding to sensor location in the vicinity of damages (see Fig. 12), the following can be observed:

- PE S-3 (i) shifts at event \#4, (ii) drifts after event \#5 and (iii) increases the amplitude (resulting from simulated daily temperature cycles) after event \#6;

- PE S-4 (i) drifts slightly after event \#1. The drift stops after event \#4 when the signal jumps, (ii) drifts after event \#5 and (iii) increases the amplitude after event \#6;

- PE S-7 exhibits no visual anomalies except slight change of the signal amplitude before and after event \#1, and after event \#6.

MFFT. MFFT processes a moving window of values from a PE signal. The selected length of the window is $1 / 3$ of the length of the reference period. The rest of the measurements in the reference period are used to determine the confidence interval for the feature used for anomaly detection. The threshold for the confidence interval is set as $+6 \sigma$, where $\sigma$ is the standard deviation during the reference period. A
A dataset encompassing $1 / 2$ of measurements before the truss was damaged forms the reference dataset. This 
Fig. 12 PE S-3, PE S-4 and PE S-7 signals [25]. Numbers in the boxes correspond to events listed in Table 1

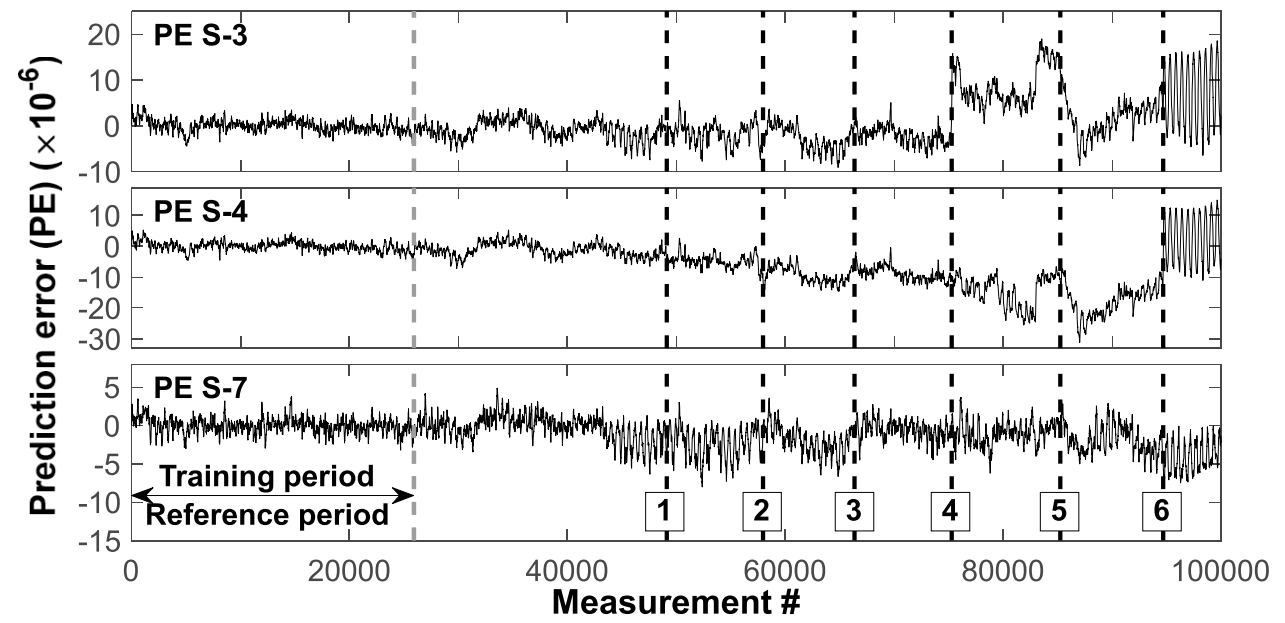

negative threshold is meaningless as the MFFT features cannot be negative. MFFT interpretations of PE S-2, PE S-3, PE S-4 and PE S-7, which are representatives of typical signal trends, are plotted in Fig. 13. From hereon these are referred to as MFFT \{sensor name\}, e.g., MFFT S-2 refers to a MFFT interpretation of PE S-2.

MFFT S-1 and MFFT S-2 follow the same trend. MFFT S-2 can be observed to exceed marginally the threshold after events \#1, \#3 and \#5, but it return to the confidence interval after events \#2, \#3 and \#4. MFFT S-3 departs permanently outside the confidence interval after event \#4. Events \#1 and \#2 are close to the sensor S-4 and these are detected by MFFT S-4. The signal exceeds the confidence interval soon after event \#1. MFFT S-5 and MFFT S-10 follow the same pattern as MFFT S-7. From MFFT S-7, damage can be detected clearly soon after event \#4, when bolts from a joint close to sensor S-7 are removed. MFFT signals computed from the other PE signals show little or no changes from their normal trends.

MPCA. PE signals are well correlated; therefore, they can be analysed as a single dataset using multivariate anomaly detection techniques. Initially, MPCA is employed on a set comprising all PE signals. The signal eigenvalues are set to 0 . The trend of a MPCA signal is expected to change significantly with a damage. Therefore, a single confidence interval is drawn for all eigenvector signals. Average $\mu$ and $\sigma$ values of all signals during the reference period
Fig. 13 MFFT S-2, MFFT S-3, MFFT S-4 and MFFT S-7 signals

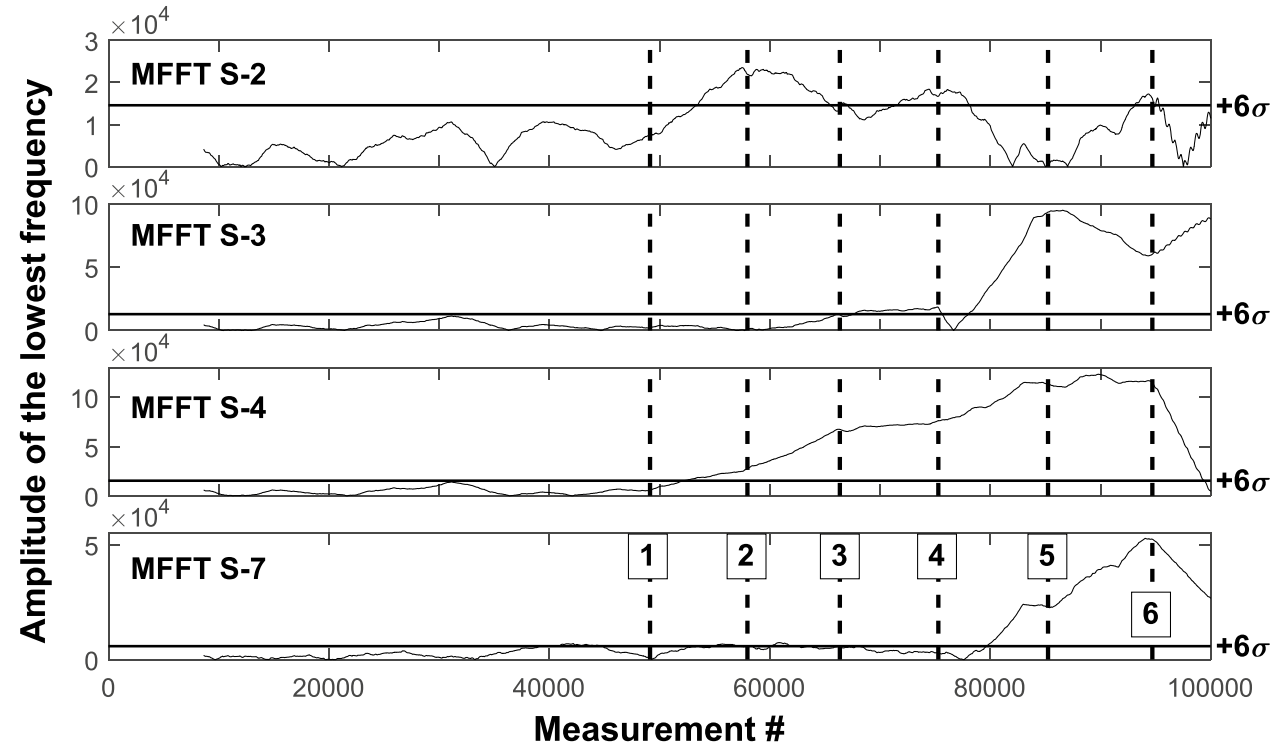


Fig. 14 Time series of the first eigenvectors computed with MPCA from PE signals: a shows eigenvectors representing S-1 to S-4 which are derived using all PE signals; $\mathbf{b}$ plots eigenvectors related to $\mathrm{S}-1, \mathrm{~S}-2$, S-4 and S-5 and c plots S-6 to $\mathrm{S}-10$, these are computed considering all but PE S-3

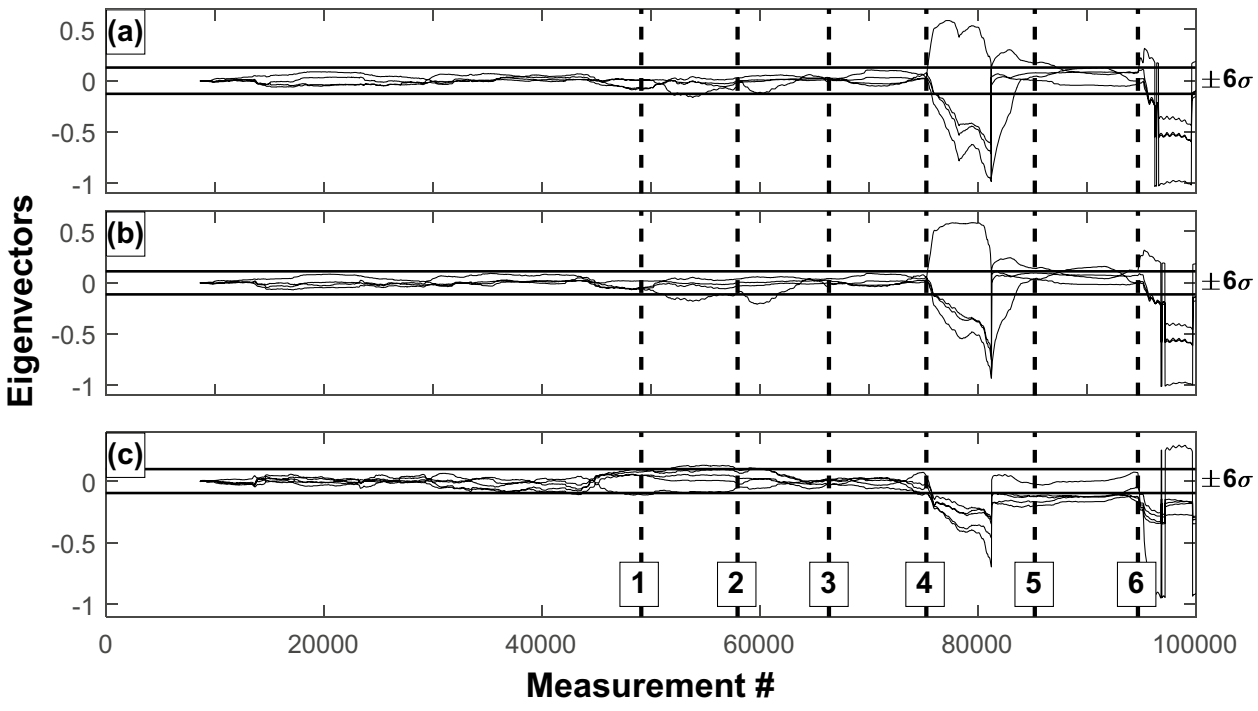

are calculated. Thresholds for the confidence interval are set to $\pm 6 \sigma$ [31]. Figure 14a shows the plot of eigenvector time histories evaluated from PE S-1, PE S-2, PE S-3 and PE S-4. A change in signal trends is noticeable soon after event \#4. The component of eigenvector signal corresponding to sensor S-3 increases, while those corresponding to other sensors drop.

MPCA is next evaluated on different sensor clusters to check if anomalous behaviour can still be detected. PE S-3, which is derived from the sensor location next to the most severely damaged joint $\mathrm{J}-1$, is excluded from the new dataset. Two clusters of PE signals are formed. One set comprises PE S-1, PE S-2, PE S-4 and PE S-5, and the other comprises PE S-6, PE S-7, PE S-8, PE S-9 and PE S-10. These correspond to sensors on the top and bottom chords, respectively. The two clusters are analysed with MPCA and evaluated eigenvectors are plotted in Fig. 14b and c, respectively. A change in signal trends can be noticed after event \#4. When boundary conditions are changed (event \#6), eigenvector signals change their trend either immediately or closely after the event. Therefore, the conclusion is that only events \#4 and \#6 are confidentially detected by MPCA when PE S-3 is included and excluded from the input set.

Cointegration. Cointegrated signals are computed from the PE signals using the process described in Sect. 2.3.2. The cointegrated signal that is ranked first is the most likely to detect anomalies, and only these are used to illustrate the technique. A cointegrated signal is initially generated for all PE signals (see Fig. 15 (top)). Thresholds for anomaly detection are specified as $\pm 3 \sigma$ [30]. The cointegrated signal shifts noticeably at event \#1, when it permanently exceeds the upper threshold, and event \#2. The sensitivity of the cointegration technique to detecting structural changes is illustrated in Fig. 15 (bottom) which shows cointegrated residuals computed from a cluster of all PE signals excluding PE S-3 and PE S-4. The cointegrated signal, while not as suggestive of anomaly events as in the case including PE S-3 and PE S-4, is still capable of detecting the majority of events. The signal drifts out of the confidence interval after event \#2, and again exceeds the upper threshold after
Fig. 15 Cointegrated signal of all PE signals (top) and all PE signals except those for sensors S-3 and S-4 (bottom)

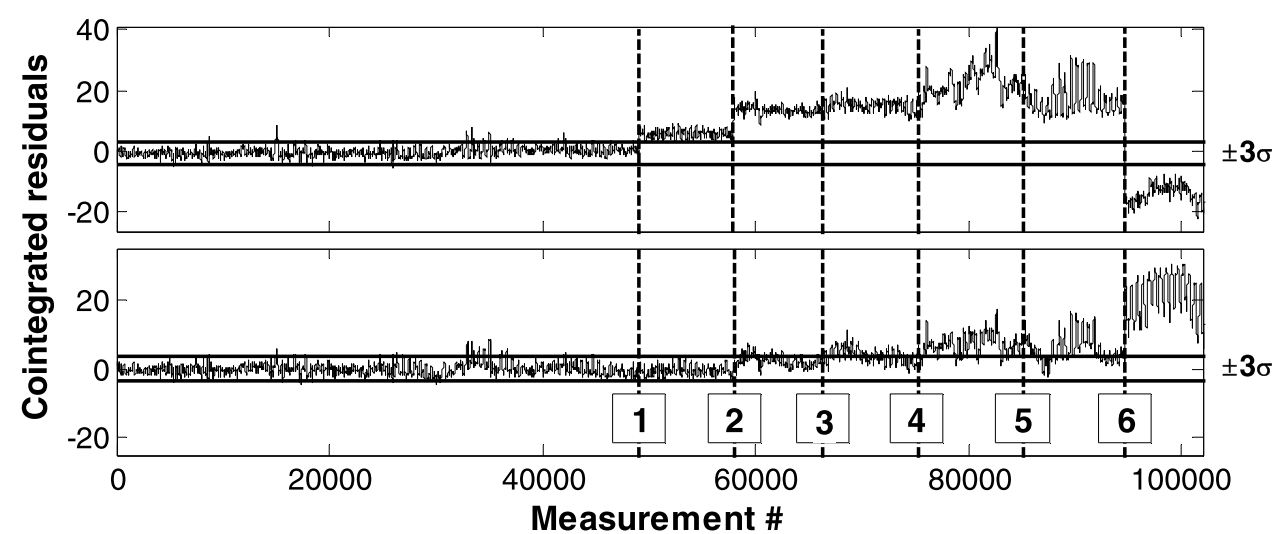


event \#4. The choice of the confidence interval is important. With $\pm 3 \sigma$ confidence interval cointegrated signal periodically exceeds the confidence interval. However, if the interval is increased $\pm 6 \sigma$, the signal would remain within the confidence bounds for periods when it marginally exceeds $\pm 3 \sigma$ limits.

SSM. SSM is used to examine all possible subtraction scenarios. The number of sensors is 10 ; hence, 45 combinations of subtracted signals are created. Subtracted signals derived from combinations of those PE signals corresponding to the sensors located away from damage exhibit no or negligible deviations from their baseline patterns. Therefore anomaly detection is demonstrated using only a few subtracted signals that are generated from PE signals of sensors close to the damaged joints. Figure 16 displays four subtracted signals which reflect the structural behaviour of the truss.

Each signal refers to a combination of two signals, e.g., subtracted signal $\mathrm{T}_{\mathrm{S} 3 \mathrm{~S} 4}$ refers to a combination of PE S-3 and PE S-4 (see Eq. 1). Combinations, which include PE $\mathrm{S}-3$ such as $\mathrm{T}_{\mathrm{S} 3 \mathrm{~S} 4}$, indicate a sudden change at event \#4. Subtracted signals, which include PE S-4 such as $\mathrm{T}_{\mathrm{S} 4 \mathrm{~S} 5}$ and $\mathrm{T}_{\mathrm{S} 4 \mathrm{~S} 8}$, depart gradually from the confidence interval after each subsequent event. Subtracted signals corresponding to a combination of sensors on elements of the truss that are not spatially close to the location of damage also diverge from their respective baseline conditions. This can be seen for $\mathrm{T}_{\mathrm{S} 4 \mathrm{~S} 8}$, which combines PE S-4 and PE S-8 corresponding to sensors S-4 and S-8. For event \#3, joint J-2 is damaged. Combining PE S-8 and PE S-9, which correspond to sensors located away from the damaged joint, but on the top chord, event \#3 is detectable from $\mathrm{T}_{\mathrm{S} 8 \mathrm{~S} 9}$ as it begins to depart outside the confidence interval.
In summary. events \#4 and \#6 can be detected with all proposed multivariate anomaly detection methodologies. Events \#1, \#2 and \#3 are not detected when PE signals are analysed using MPCA. These events, however, can be detected using cointegration and SSM techniques. Therefore, cointegration can detect changes in structural behaviour, and further investigations, for example with SSM or/and MFFT, can reveal the location of damage.

\subsubsection{Anomaly detection from response measurements}

In this section, response measurements are directly analysed using the four anomaly detection techniques while completely ignoring temperature measurements, which is the approach that has been adopted by most researchers in SHM. The purpose is to investigate if there is an improvement in anomaly detection performance by accounting for temperature effects as done in this research through using the RBTRP methodology.

The response measurements are analysed using the same values for parameters such as the reference period and the size of the moving window, as used for the interpretation of $\mathrm{PE}$ signals in Sect. 4.1.1. The training period and the length of the moving window are selected to be $1 / 3$ of the reference period. The final $2 / 3$ of measurements in the reference period is used to determine the confidence interval.

$\underline{M F F T}$. MFFT signals computed from response (strain) measurements offer no support for detecting anomalous structural behaviour, and are, hence, not shown here.

MPCA. Events can be detected, when clusters of strain signals are analysed with MPCA. Eigenvectors, which are related to all signals except those of sensors S-5 and S-10,
Fig. 16 Subtracted signals $\mathrm{T}_{\mathrm{S} 3 \mathrm{~S} 4}, \mathrm{~T}_{\mathrm{S} 4 \mathrm{~S} 5}, \mathrm{~T}_{\mathrm{S} 4 \mathrm{~S} 8}$ and $\mathrm{T}_{\mathrm{S} 4 \mathrm{~S} 9}$ generated with SSM [25]

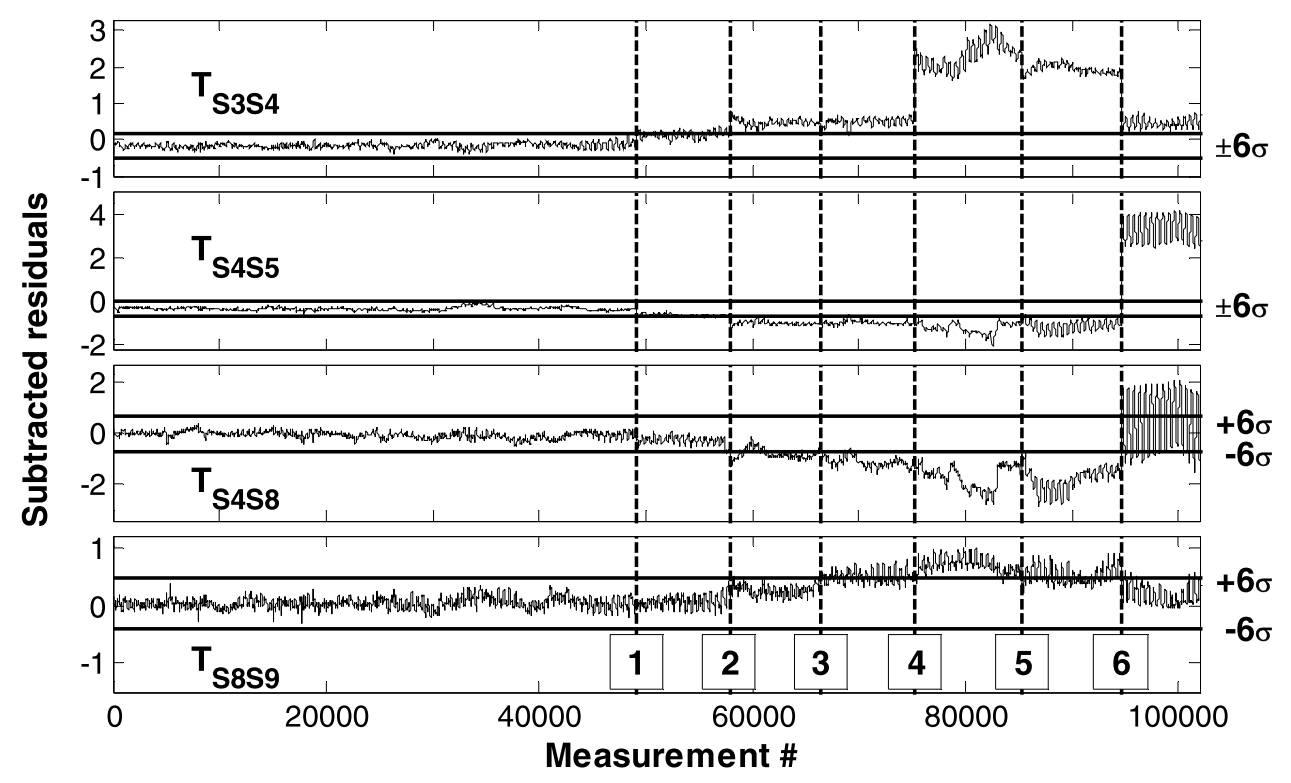


change their pattern after event \#6. Events \#1, \#2 and \#3 are not revealed with MPCA. Figure 17 illustrates an eigenvector signal related to sensor location S-3. Shortly after event \#1, the eigenvector signal marginally and temporarily exceeds the confidence interval, which is set to $\pm 3 \sigma$. This change alone may be unreliable to state whether there is a change in structural performance. After event \#4 (after measurement \#80,000), the eigenvector signal exceeds the upper threshold and returns to the confidence interval after event \#5 when the truss is repaired. The author hypothesises that the signal would have remained outside the confidence interval, if the joints were not repaired. Lastly, the change of boundary conditions (event \#6) is also immediately reflected in the eigenvector signals of response measurements (see Fig. 17). These observations are similar to those made for MPCA results on PE signals (illustrated in Fig. 14).

Cointegration. The cointegrated signal of response measurements is plotted in Fig. 18. The signal is stationary during the reference period and prior to event \#1. The signal shifts immediately after events \#1, \#2, \#4 and \#6. These events have changed the performance of the structure and can clearly be identified in the cointegrated signal. The signal, however, does not return to its original pattern shown during the reference period after the truss is repaired (event \#5). Thus, applying cointegration directly on measured response can indicate anomaly events. However, the reliability of anomaly detection is better when analysing PE signals after using the RBTRP methodology (see Fig. 15) since the shifts from anomaly events are much more pronounced and therefore easier to identify.

$\underline{\text { SSM. }}$. Subtracted signals can indicate events \#4 and \#6. Subtracted signal $\mathrm{T}_{\mathrm{S} 3 \mathrm{~S} 4}$ computed from response measured by sensors S-3 and S-4 that are located on the bottom chord shift abruptly after event \#4 (Fig. 19). All subtracted signals are capable of indicating event \#6 by showing an abrupt shift, such as can be observed in $\mathrm{T}_{\mathrm{S} 4 \mathrm{~S} 5}$, when boundary conditions are changed (event \#6). In contrast, subtracted signals computed from PE signals are able to detect most of the events with a higher degree of confidence.
Fig. 17 Time series of the first eigenvectors related to sensor S-3 computed with MPCA scenario $\mathrm{X}$

Fig. 18 Cointegrated signal of all strain measurements for scenario $\mathrm{X}$ from all strain measurements for
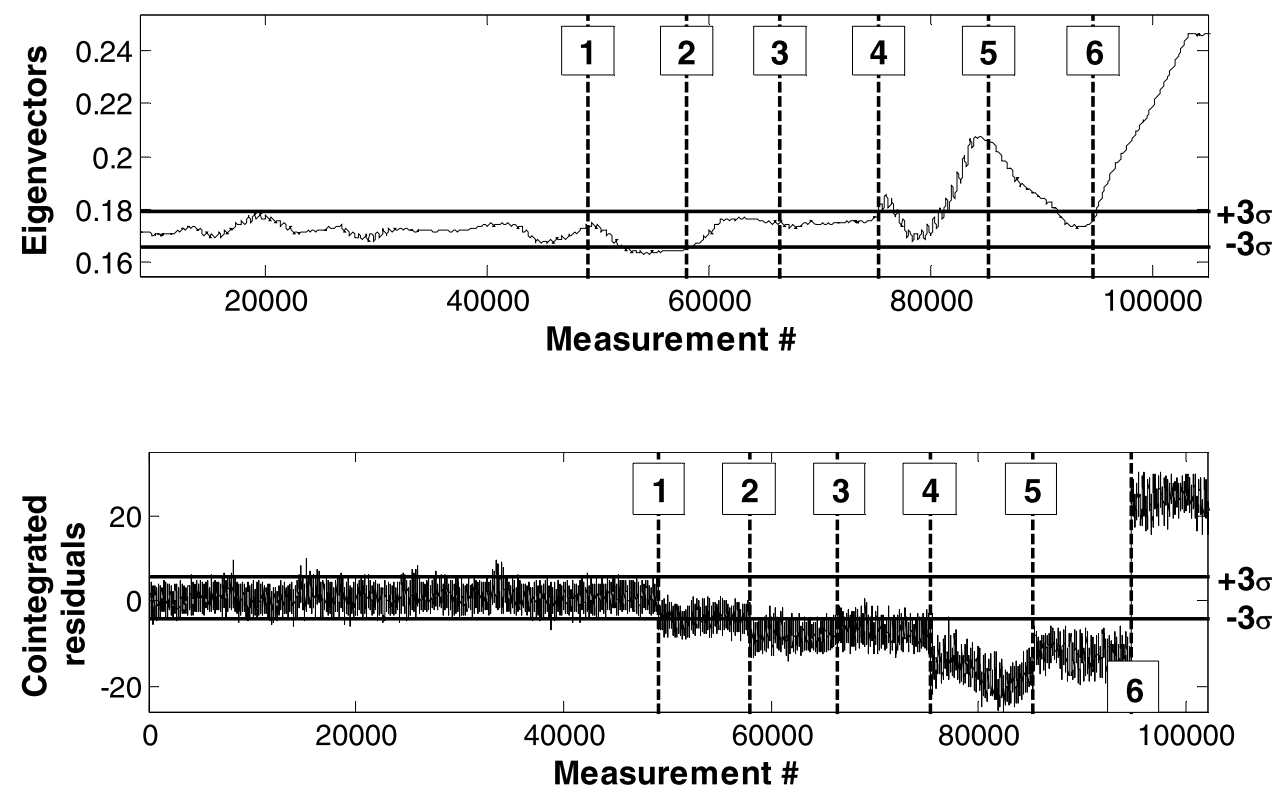

Fig. 19 Subtracted signals $\mathrm{T}_{\mathrm{S} 3 \mathrm{~S} 4}$ and $\mathrm{T}_{\mathrm{S} 4 \mathrm{~S} 5}$ generated with SSM from strain measurements

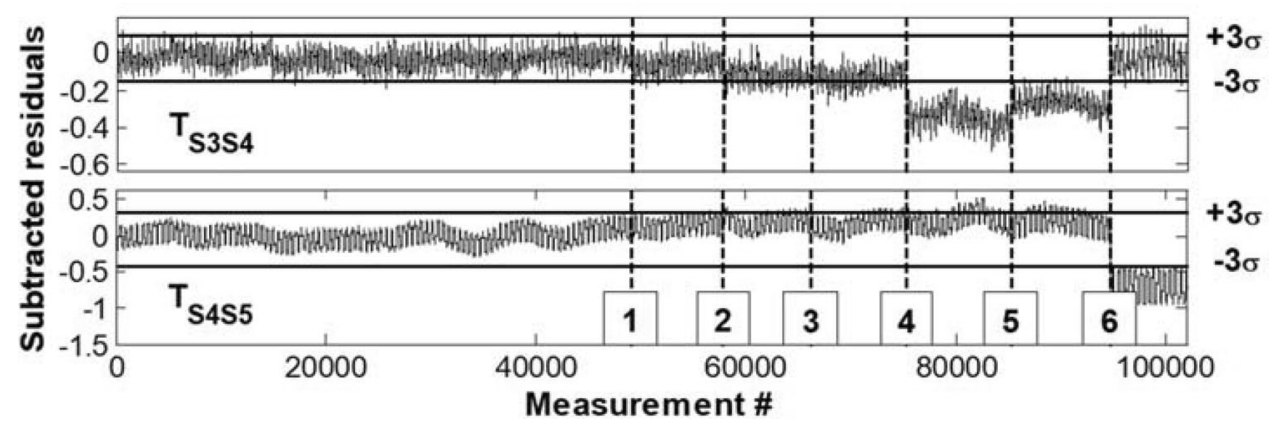


Table 3 Summary of anomaly detection results

\begin{tabular}{llllllll}
\hline Interpretation approach & $\begin{array}{l}\text { Anomaly detection } \\
\text { technique }\end{array}$ & Event & & & & & \\
\cline { 2 - 8 } & & $\# 1$ & $\# 2$ & $\# 3$ & $\# 4$ & $\# 5$ & $\# 6$ \\
\hline TB-MI & MFFT & Yes & Yes & No & Yes & N/A & N/A \\
& MPCA & No & No & No & No & N/A & N/A \\
& Cointegration & No & No & No & No & N/A & N/A \\
& SSM & Yes & Yes & Yes & Yes & N/A & N/A \\
& MFFT & No & No & No & No & N/A & N/A \\
Response only & MPCA & No & No & No & No & N/A & N/A \\
& Cointegration & No & No & No & No & N/A & N/A \\
& SSM & No & No & No & $Y e s$ & N/A & N/A \\
\hline
\end{tabular}

Anomaly detection level: italic indicate definitive, roman indicate semi-definitive and bold indicate not detectible

Damage location detected: yes, no, not applicable (N/A)

\subsubsection{Summary}

Table 3 summarises the results from the TB-MI and response-only approaches for the four selected anomaly detection techniques. A traffic light visualisation approach is chosen. Green colour indicates that there is a definitive evidence of a change in the signal detecting an anomaly event; yellow suggests that the evidence is semi-definitive; and red indicates that the event is not detectible. If the damage location is also detected, the event field has a 'yes' in it. An example of a case where there is definitive evidence of damage and a clear indication of its location is found in Fig. 13 where signal MFFT S-4 exceeds the damage indicating threshold. Cases with semi-definitive evidence of damage include MFFT S-3 in Fig. 13, and $\mathrm{T}_{\mathrm{S} 3 \mathrm{~S} 4}$ and $\mathrm{T}_{\mathrm{S} 4 \mathrm{~S} 5}$ in Fig. 16 after event \#3, where signals are close to the limit of the confidence interval. Identifying damage location with multi-variate techniques is difficult when signals are not split into clusters, therefore damage locations are not detected. Overall, the TB-MI approach provides better damage detection and localisation than the response-only approach.

\subsection{NPL footbridge}

\subsubsection{Thermal response predictions}

The implementation of the RBTRP methodology for the generation of regression models for thermal response prediction of the NPL footbridge is briefly discussed here. Detailed information can be found in the authors' previous work [28]. The model generation can be a computationally intensive task when analysing large datasets. Therefore, the first step is to find the optimal range of input parameters to the model training using a down-sampled dataset from the reference period. The reference period should encompass as much of the anticipated variability in measurements as possible, e.g. one seasonal cycle. Measurements from the first year of monitoring, which constitute a total of 94,000 measurements, are selected.

A sampling frequency of $6.5 \times 10^{-6} \mathrm{~Hz}$ is selected to identify the range of desirable input parameters to the regression models. The two parameters are the thermal inertia parameter $j$ and the number of principal components (PCs). The input to the regression models is composed of both current $\left(D_{i}\right)$ and former temperature $\left(D_{i-j}\right)$ measurements that have been transformed into PC space, where $i$ refers to the most recent measurement and $j$ to a previous measurement set collected $j$ time-steps before $i$ [28]. As there are ten temperature sensors on the bridge, the number of PCs range from 1 to 10. The first half of the PCs is sufficient to achieve good response predictions [25]. Support vector regression is chosen for the model generation. Mean PEs for regression models are plotted versus the number of PCs and the parameter $j$ for sensors TL- 1 and TL-4 in Fig. 20. The desired values of the two parameters are defined from these plots. Then, model training is repeated with a larger set of training data than in the first step using the number of PCs and the parameter $j$ that are found to give the lowest PEs.

Reasonably accurate regression models are computed using the available information, which contains imperfections in the dataset such as the lack of a reference period free of anomaly events. Resulting PE signals are shown in Fig. 21. Table 4 provides the statistics of the PEs for all sensor locations. PEs are expressed in terms of the root mean square error (RMSE) and the percentage of the range of tilts measured during the reference period. Overall, the PEs are close to $10 \%$ or smaller. As structural behaviour may have been altered after events listed in Table 2 and only limited knowledge of temperature distribution is available from measurements, the generated regression models are satisfactory. 

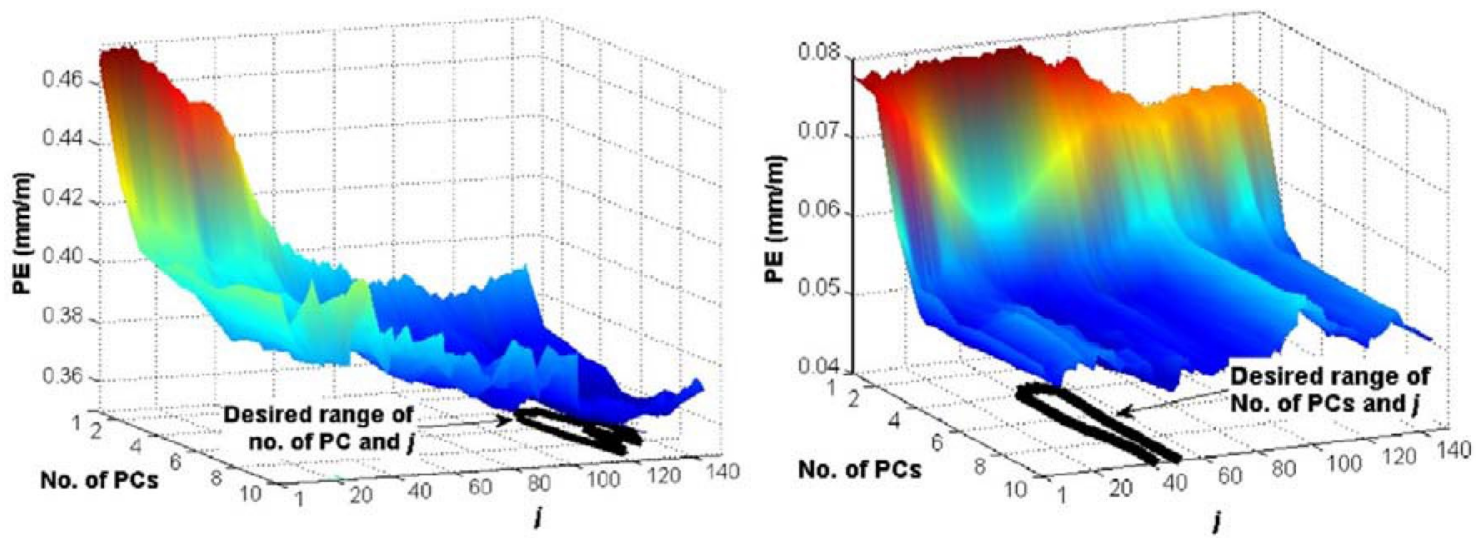

Fig. 20 Tilt prediction error $(\mathrm{mm} / \mathrm{m})$ versus the number of PCs and thermal inertia parameter $j$ for prediction models computed for sensors TL-1 (left) and TL-4 (right)

Fig. 21 Prediction error (PE) signals for all sensor locations. Numbers in boxes represent events listed in Table 2

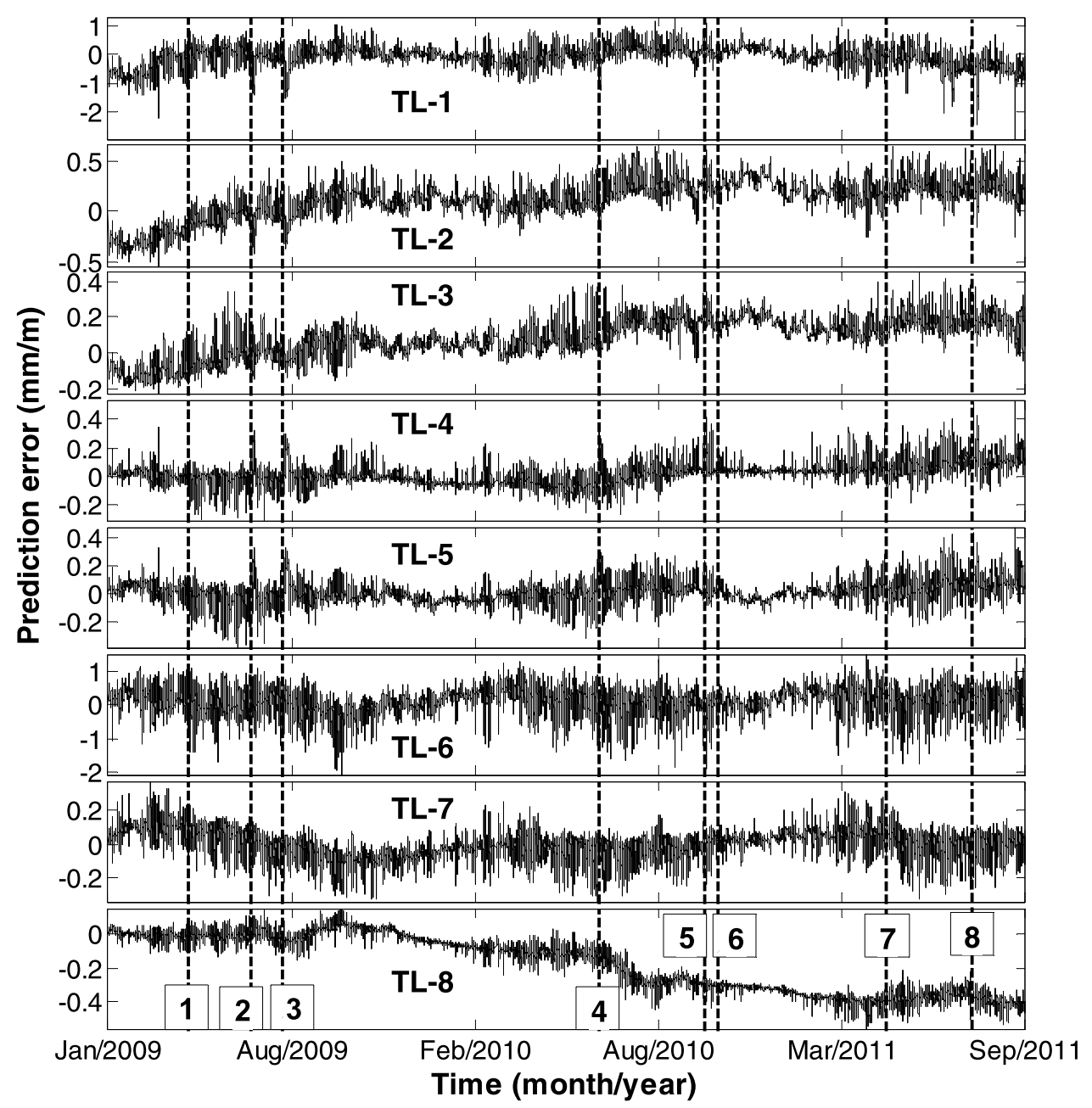

\subsubsection{Anomaly detection}

PE signals are first visually examined for patterns and any imperfections. Of all the signals, PE TL- 8 alone shows a different pattern. Measurements from sensor TL-8 do not resemble typical seasonal temperature patterns (see Fig. 10). In fact, the measurements show two noticeable drifts that commence at the following times: 
Table 4 Tilt prediction accuracy for the reference period

\begin{tabular}{lllc}
\hline Sensor name & $\begin{array}{l}\text { The range of tilts for } \\
\text { the model training } \\
(\mathrm{mm} / \mathrm{m})\end{array}$ & RMSE $(\mathrm{mm} / \mathrm{m})$ & RMSE (\%) \\
\hline TL-1 & 3.27 & 0.353 & $10.8 \%$ \\
TL-2 & 0.91 & 0.165 & $18.3 \%$ \\
TL-3 & 0.55 & 0.047 & $14.5 \%$ \\
TL-4 & 0.95 & 0.079 & $4.9 \%$ \\
TL-5 & 1.12 & 0.058 & $5.1 \%$ \\
TL-6 & 4.00 & 0.292 & $7.3 \%$ \\
TL-7 & 0.63 & 0.086 & $13.7 \%$ \\
TL-8 & 0.36 & 0.032 & $8.8 \%$ \\
\hline
\end{tabular}

- the beginning of winter 2010 and lasting until spring 2010;

- shortly after event \#4.

Consequently, PE TL-8 is also exhibiting a similar pattern, and also has high values due to the large prediction errors, particularly after event \#4 (Fig. 21).

A few of the events can be identified directly from the PE signals due to prior knowledge of the event history. Spikes at events \#2 and \#3 are discernible in PE TL-1, PE TL-4 and PE TL-5. However, in general, all the events cannot be identified by visually examining the PE signals. The PE signals are, therefore, processed for anomaly events using MFFT, MPCA, cointegration and SSM.

PE TL-1 drifts slightly from the beginning of the monitoring until event \#1. Such signal behaviour is not observed in PE TL-6 (sensor TL-6 is located on the other cantilever of the footbridge). A drift in PE TL-7 is discernible after event \#1. This drift lasts for several months and the signal stabilises at the end of the reference period. This can be determined only after the PE signals are analysed for anomalies.

$\underline{M F F T}$. The length of the moving window is set to 4 months, which is $1 / 3$ of the length of the reference period. The later
$2 / 3$ of the measurements in the reference period are used to derive the confidence interval. Each MFFT signal is different and no common trends are noticeable. Some MFFT signals temporarily exceed the confidence interval, the others stay within the specified limits. Plots of MFFT TL-3 and MFFT TL-8 are shown in Fig. 22. MFFT TL-3 departs the confidence interval after event \#4. However, MFFT TL-8 exceeds the confidence interval soon after the end of the reference period, before February 2010. During this period, which is between events \#3 and \#4, no activity affecting the performance of the footbridge took place.

MPCA. PE signals of all tilt sensors are used as input to MPCA. Initially, the length of the moving window is set to be the same as for MFFT, i.e. 4 months. No anomaly events are revealed in eigenvector signals (Fig. 23 (top)). However, many of the events are indicated by spikes in PE signals as discussed earlier (see Fig. 10). The length of the moving window is, therefore, gradually reduced to investigate if these events can be detected from analysis of PE signals using shorter lengths for the moving windows. The plot of the component from the first eigenvector signal corresponding to sensor TL-1 computed using a moving window length of 30 days is also shown in Fig. 23 (bottom). The eigenvector values jump immediately after events \#1, \#2, $\# 3$, \#4 and \#8. Events \#5, \#6 and \#7 are not detected. These events may have had little effect on the overall structural performance. Also, the eigenvector signal jumps not at event \#8 but slightly after. This takes place on July 7, 2011. On this day, static load tests were carried out, which may have amplified the response due to the permanent damage created during event \#8.

Cointegration. All PE signals are analysed using cointegration. The cointegrated signal is plotted in Fig. 24. The signal departs from the confidence interval after event \#4. This closely reflects the trend of PE TL-8, which may indicate that the event happened close to sensor TL-8. However, the cointegrated signal cannot offer support in detecting

Fig. 22 MFFT TL-3 and MFFT TL-8

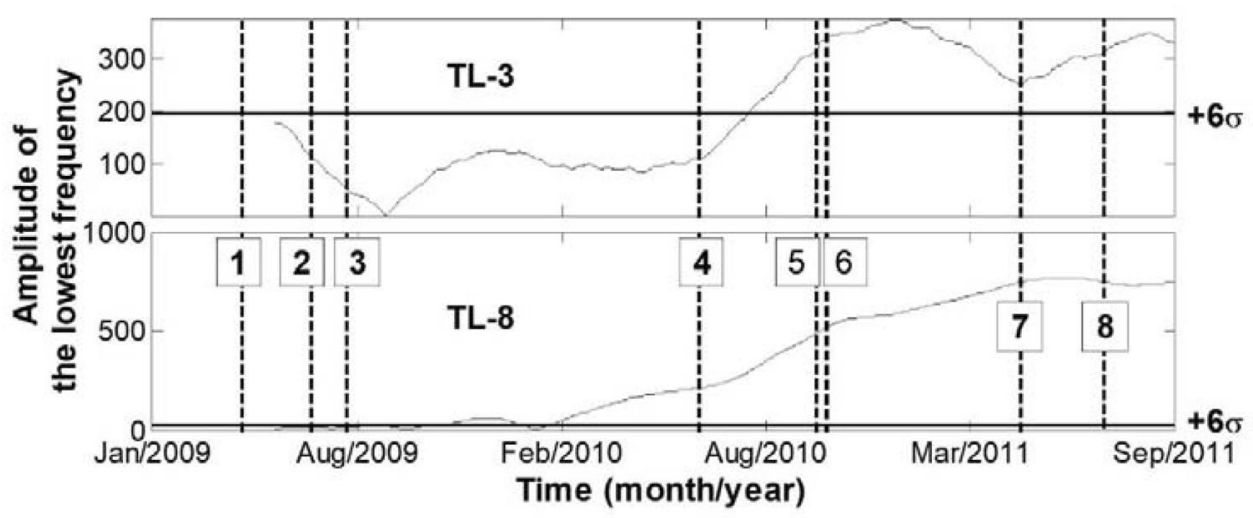


the location of the events. All other events appear to create small drifts in the cointegrated signal. However, none of the events other than event \#4 are reliably detected using the cointegrated signal. This is in agreement with a previous study by Worden et al. [37], where they demonstrated that anomalous events from the NPL Footbridge can be detected when analysing all response measurements using the cointegration approach.

SSM. The interpretation of PE signals with SSM provides results similar to those obtained using MPCA and cointegration. Subtracted signals, which are derived using PE TL-8, exceed the confidence interval soon after event \#4. As an example, $\mathrm{T}_{\mathrm{TL} 1 \mathrm{TL} 8}$ is plotted in Fig. 25. It resembles the cointegrated signal shown in Fig. 24. In addition to detecting event \#4, these results also indicate that the event must have occurred close to TL-8. Subtracted signals, generated from combinations including PE TL-4 and PE TL-5 have shifts right after at events \#2, \#3 and \#4. However, the shifts due to these events, which have short timescales, can be noticed only when the signals are examined closely as illustrated using $\mathrm{T}_{\text {TL1TL5 }}$ in Fig. 26. The results also indicate that events \#2, \#3 and \#4 must have occurred near sensors TL-4 and TL-5.

\subsection{Discussion}

$\pm 3 \sigma$ confidence intervals are more sensitive to changes in damage sensitive features than $\pm 6 \sigma$ intervals. $\pm 3 \sigma$ intervals are appropriate when analysing signals which are fairly
Fig. 23 Time series of the first computed with MPCA from all PEs with. The length of the moving window is four months and 30 days for the top and bottom plots, respectively eigenvectors related to TL-1
Fig. 24 Cointegrated signal generated from all PE signals
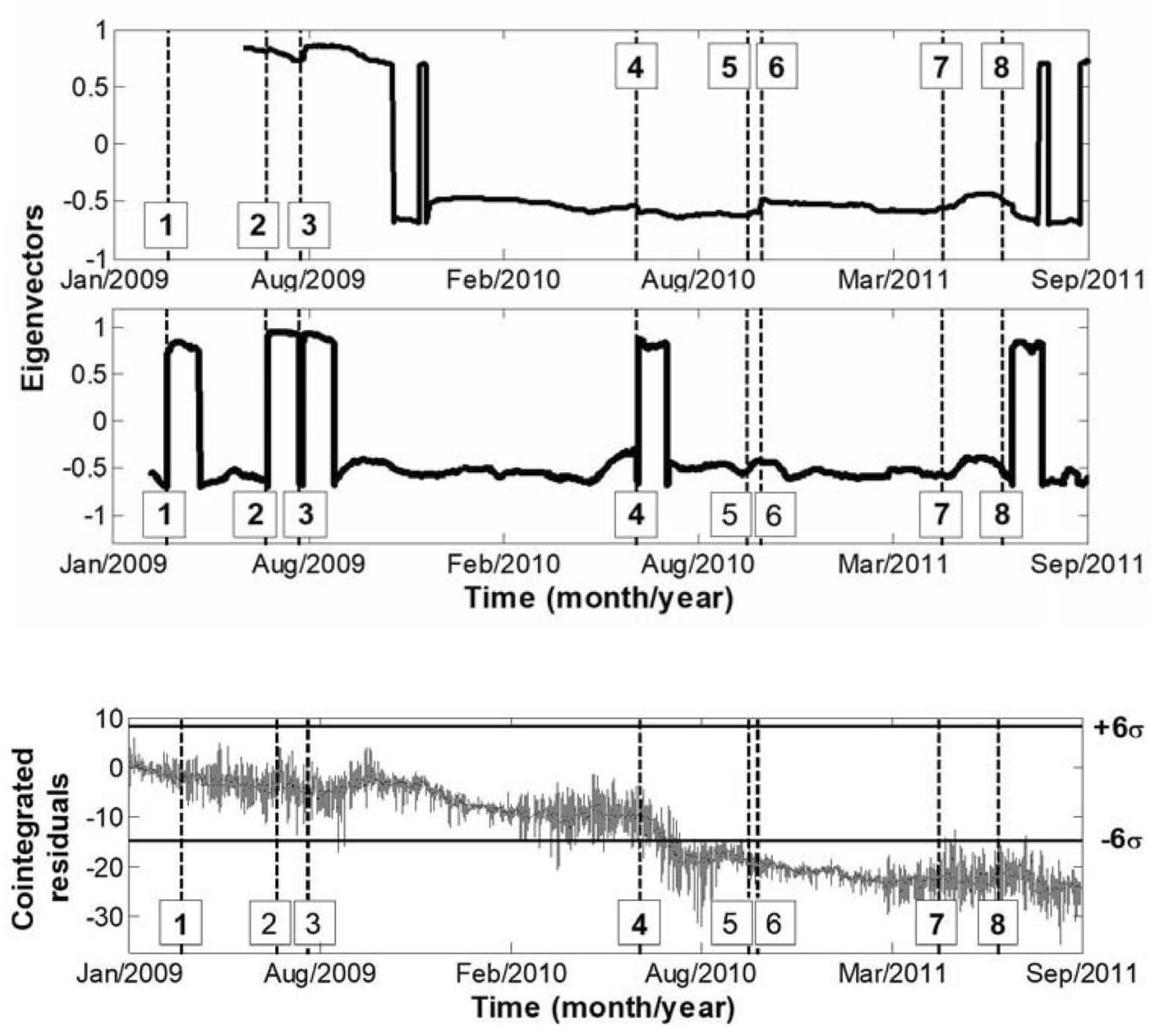

Fig. $25 \mathrm{~T}_{\mathrm{TL} 1 \mathrm{TL} 8}$ generated with SSM

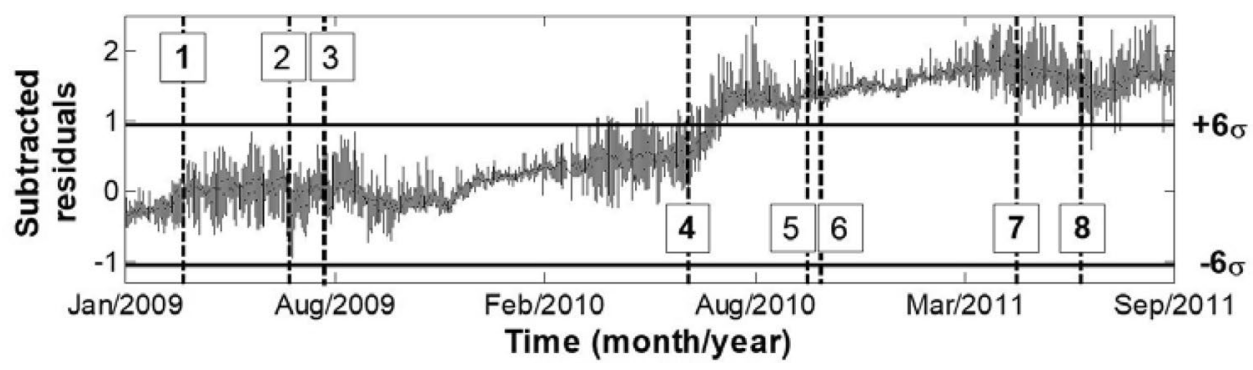


Fig. $26 \mathrm{~T}_{\mathrm{TL} 1 \mathrm{TL} 5}$ generated with SSM. Plot at top shows the signal for the full measurement history, while those on the bottom are closer views near events \#2, \#3 and \#4
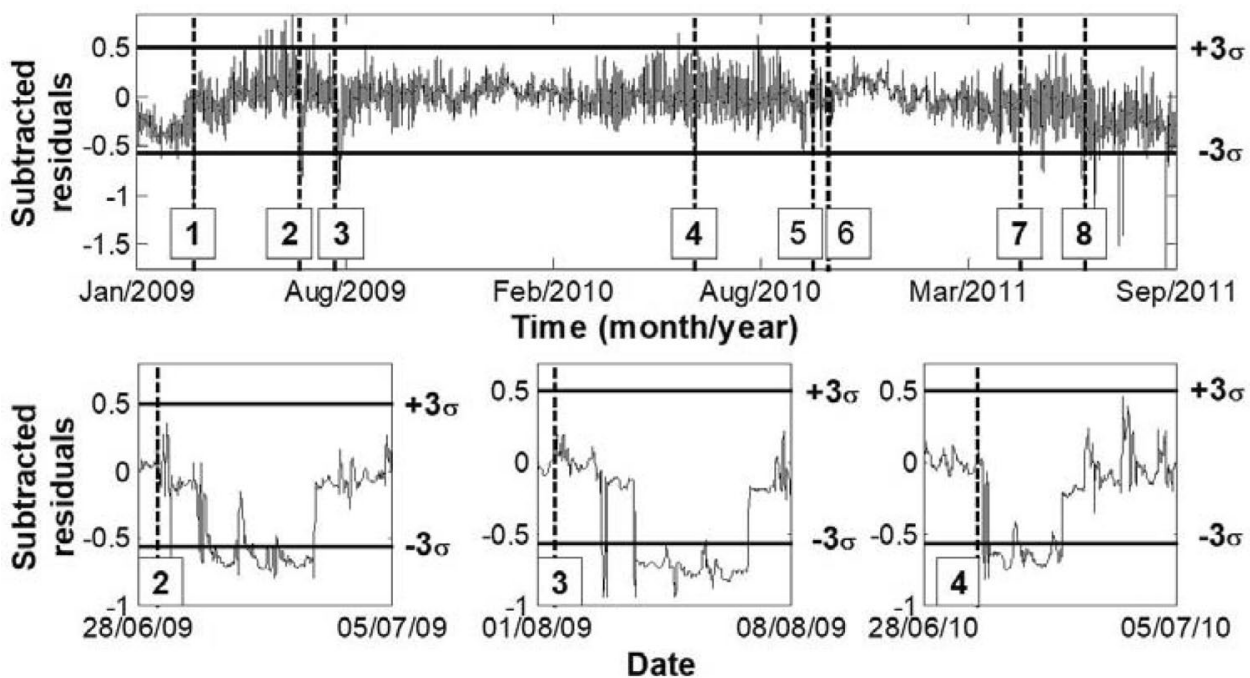

stationary in nature such as cointegrated residuals (see Fig. 15) and when inspecting signals for short anomaly periods (see Fig. 26). As shown in the laboratory truss example, the analysed signal deviates further away from the confidence interval when the severity of damage aggravates.

The case study of the laboratory truss demonstrates that a single method does not detect all damage events. However, often there is at least one method that detects each damage event. Multivariate signal analyses are useful for detection of damage, however, in order to locate damage, signal clusters have to be created and analysed. Univariate signal analyses, on the other hand, are good for suggesting damage location; however, they may also be sensitive to changes in signal that do not indicate anomalies and thus raise false alarms. Therefore combining results from a range of signal processing techniques has the potential to significantly increase the confidence in anomaly detection. The results from all methods can be combined to increase the reliability of decisions and reduce false positives. This is a topic for future study and an example is demonstrated in the next paragraph.

A combination of two anomaly detection methods is observed to increase the damage detectability [31]. For example, the MFFT method is used to analyse $\mathrm{T}_{\mathrm{S} 3 \mathrm{~S} 4}$ (see Fig. 27) from the laboratory truss. The new signal exceeds slightly $+6 \sigma$ threshold during the period when the truss is not damage. This, however, is not discernible in the plot due to the large range of the signal after the damage. After damaging joint J-1 (event \#1), a significant change is discernible in the signal trend. Continuous signal departures away from the confidence interval are observed after event \#1, \#2 and 4. These are events when damage is made close to sensor locations S-3 and S-4. The signal becomes stationary after event \#3 when joint $\mathrm{J}-2$ is damaged. This joint is on the top chord.

While the TB-MI approach has shown promising results, further investigation into anomaly classification is required to scale it up for practical applications. Anomaly detection is currently based on certain metrics exceeding pre-defined thresholds. However, to avoid false positives, these thresholds may have to be flexible to account for long-term shifts in response due to expected benign changes in material behaviour such as from creep.

Lastly, the current study has focused primarily on temperature-induced response measurements. The structural response to other forms of loading such as wind and vehicle may also be treated in a similar manner. Knowledge of wind and vehicle loading can enable the prediction of related response, which upon removal from measured response can improve significantly anomaly detection. This is a natural next step to the work presented in this paper.
Fig. 27 MFFT interpretation of $T_{S 3 S 4}$ signal

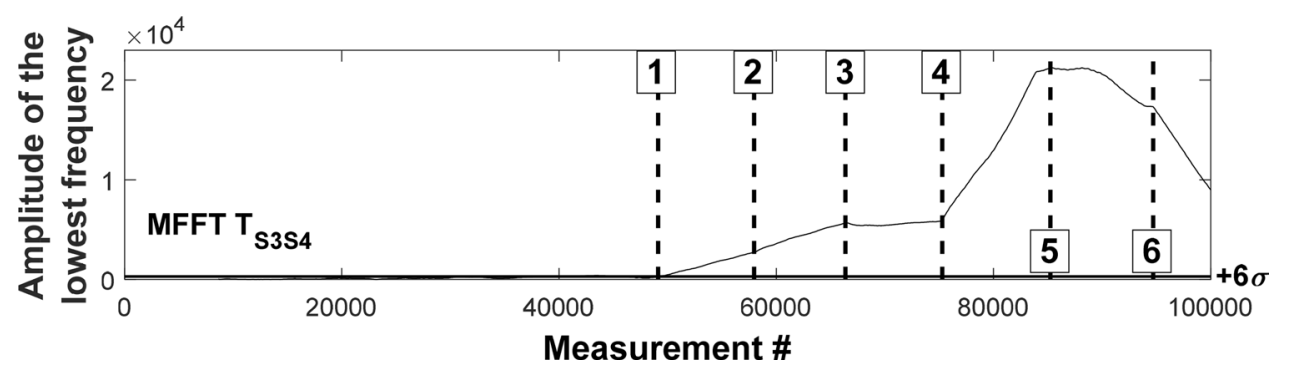




\section{Conclusions}

Four anomaly detection techniques are compared with respect to their performance within the TB-MI approach and on response measurements only using measurements from a laboratory truss and a full-scale footbridge. The results show that anomaly detection is not easy. The application of multiple anomaly detection techniques is necessary to determine each type of event (damage). The following conclusions are drawn:

- The TB-MI approach provides better anomaly detection and localisation results than the response-only analysis. However, the analysis of response only does not require distributed temperature measurements, which are mandatory for the application of the TB-MI approach.

- Cointegration and signal subtraction method are more robust in detecting anomalies than moving principal component analysis and moving fast Fourier transform. Cointegration can only be used to detect the occurrence of an anomaly event while SSM can help reveal the location of damage.

- Using moving windows of short lengths in MPCA can detect temporary anomaly events such as abnormal shortterm loadings. These events can also be detected while having a closer look at signals generated with SSM. Thus, an ensemble of methodologies may be required for faster, and more robust and reliable detection of both temporary anomalous behaviour (e.g. previously unseen loading) and permanent damage.

- The global structural behaviour of a bridge changes if an element critical to the load path is damaged. The TB-MI approach can detect the global damage, if its extent is significant, even using signals from sensors located away from the location of damage.

- The selection of an appropriate confidence interval is challenging. $\pm 6 \sigma$ confidence intervals may not reveal short-term anomaly events, but $\pm 3 \sigma$ intervals may result in false negatives. The truss experiment demonstrates that interpreted signals change significantly after damages are introduced. Combined methods such as SSM and MFFT can be considered to improve the performance of the damage detection techniques.

Acknowledgements Authors would like to express their gratitude to Dr. Elena Barton from National Physical Laboratory for providing data of the NPL footbridge.

\section{Compliance with ethical standards}

Conflict of interest The authors declare that they have no conflict of interest.
Open Access This article is licensed under a Creative Commons Attribution 4.0 International License, which permits use, sharing, adaptation, distribution and reproduction in any medium or format, as long as you give appropriate credit to the original author(s) and the source, provide a link to the Creative Commons licence, and indicate if changes were made. The images or other third party material in this article are included in the article's Creative Commons licence, unless indicated otherwise in a credit line to the material. If material is not included in the article's Creative Commons licence and your intended use is not permitted by statutory regulation or exceeds the permitted use, you will need to obtain permission directly from the copyright holder. To view a copy of this licence, visit http://creativecommons.org/licenses/by/4.0/.

\section{References}

1. Aktan AE, Brownjohn JMW (2013) Structural identification: opportunities and challenges. J Struct Eng 139:1639-1647. https ://doi.org/10.1061/(ASCE)ST.1943-541X.0000723

2. Li H, Ou J (2016) The state of the art in structural health monitoring of cable-stayed bridges. J Civ Struct Heal Monit 6:43-67. https://doi.org/10.1007/s13349-015-0115-x

3. Sazonov E, Li H, Curry D, Pillay P (2009) Self-powered sensors for monitoring of highway bridges. Sens J IEEE 9:1422-1429

4. Worden K, Cross EJ, Brownjohn JMW (2013) Switching response surface models for structural health monitoring of bridges. In: Surrogate-based modeling and optimization. Springer, Berlin, pp 337-358

5. Abdel-Jaber H, Glisic B (2016) Systematic method for the validation of long-term temperature measurements. Smart Mater Struct 25:125025

6. Cunha A, Caetano E, Magalhães F, Moutinho C (2013) Recent perspectives in dynamic testing and monitoring of bridges. Struct Control Heal Monit 20:853-877. https://doi.org/10.1002/ stc

7. Cross EJ, Koo KY, Brownjohn JMW, Worden K (2013) Longterm monitoring and data analysis of the Tamar Bridge. Mech Syst Signal Process 35:16-34. https://doi.org/10.1016/j.ymssp .2012.08.026

8. Kromanis R, Kripakaran P, Harvey B (2016) Long-term structural health monitoring of the Cleddau bridge: evaluation of quasi-static temperature effects on bearing movements. Struct Infrastruct Eng. https://doi.org/10.1080/15732479.2015.11171 13

9. Sawicki B, Brühwiler E (2020) Long-term strain measurements of traffic and temperature effects on an RC bridge deck slab strengthened with an R-UHPFRC layer. J Civ Struct Heal Monit 10:333-344. https://doi.org/10.1007/s13349-020-00387-3

10. Hua XG, Ni YQ, Ko JM, Wong KY (2007) Modeling of temperature-frequency correlation using combined principal component analysis and support vector regression technique. J Comput Civ Eng 21:122-135

11. Murphy B, Yarnold M (2018) Temperature-driven structural identification of a steel girder bridge with an integral abutment. Eng Struct 155:209-221. https://doi.org/10.1016/j.engst ruct.2017.10.074

12. Potgieter IC, Gamble WL (1989) Nonlinear temperature distributions in bridges at different locations in the United States. PCI J 34:80-103

13. Catbas FN, Kijewski-Correa T, Aktan AE (2011) Structural identification (St-Id) of constructed facilities: approaches, methods and technologies for effective practice of St-Id. Am Soc Civ Eng. https ://doi.org/10.1061/9780784411971 
14. Noël JP, Kerschen G (2017) Nonlinear system identification in structural dynamics: 10 more years of progress. Mech Syst Signal Process 83:2-35. https://doi.org/10.1016/j.ymssp.2016.07.020

15. Rytter A (1993) Vibrational based inspection of civil engineering structures. Diss. Dept. of Building Technology and Structural Engineering, Aalborg University. https://www.forskningsdatab asen.dk/en/catalog/2389379256

16. Worden K, Dulieu-Barton JM (2004) An overview of intelligent fault detection in systems and structures. Struct Heal Monit 3:85-98

17. Goulet JA, Kripakaran P, Smith IFC (2010) Multimodel structural performance monitoring. J Struct Eng 136:1309-1318

18. Cavadas F, Smith IFC, Figueiras J (2013) Damage detection using data-driven methods applied to moving-load responses. Mech Syst Signal Process 39:409-425

19. Farreras-Alcover I, Chryssanthopoulos MK, Andersen JE (2017) Data-based models for fatigue reliability of orthotropic steel bridge decks based on temperature, traffic and strain monitoring. Int J Fatigue 95:104-119. https://doi.org/10.1016/j.ijfat igue.2016.09.019

20. Weinstein JC, Sanayei M, Brenner BR (2018) Bridge damage identification using artificial neural networks. J Bridg Eng 23:04018084. https://doi.org/10.1061/(ASCE)BE.19435592.0001302

21. Ruffels A, Gonzalez I, Karoumi R (2020) Model-free damage detection of a laboratory bridge using artificial neural networks. $\mathrm{J}$ Civ Struct Heal Monit 10:183-195. https://doi.org/10.1007/s1334 9-019-00375-2

22. Posenato D, Kripakaran P, Inaudi D, Smith IFC (2010) Methodologies for model-free data interpretation of civil engineering structures. Comput Struct 88:467-482

23. Kromanis R, Kripakaran P (2013) Support vector regression for anomaly detection from measurement histories. Adv Eng Informatics 27:486-495. https://doi.org/10.1016/j.aei.2013.03.002

24. Kromanis R, Kripakaran P (2017) Data-driven approaches for measurement interpretation: analysing integrated thermal and vehicular response in bridge structural health monitoring. Adv Eng Informatics 34:46-59. https://doi.org/10.1016/j. aei.2017.09.002

25. Kromanis R, Kripakaran P (2016) SHM of bridges: characterising thermal response and detecting anomaly events using a temperature-based measurement interpretation approach. J Civ Struct Heal Monit 6:237-254. https://doi.org/10.1007/s13349-016-0161-z

26. Yarnold MT, Moon FL, Aktan AE (2015) Temperature-based structural identification of long-span bridges. J Struct Eng. https ://doi.org/10.1061/(ASCE)ST.1943-541X.0001270

27. Cross EJ, Worden K, Chen Q (2011) Cointegration: a novel approach for the removal of environmental trends in structural health monitoring data. Proc R Soc A Math Phys Eng Sci 467:2712-2732. https://doi.org/10.1098/rspa.2011.0023
28. Kromanis R, Kripakaran P (2014) Predicting thermal response of bridges using regression models derived from measurement histories. Comput Struct 136:64-77. https://doi.org/10.1016/j. compstruc.2014.01.026

29. Gul M, Catbas FN (2011) Structural health monitoring and damage assessment using a novel time series analysis methodology with sensor clustering. J Sound Vib 330:1196-1210. https://doi. org/10.1016/j.jsv.2010.09.024

30. Shi H, Worden K, Cross EJ (2018) A regime-switching cointegration approach for removing environmental and operational variations in structural health monitoring. Mech Syst Signal Process 103:381-397. https://doi.org/10.1016/j.ymssp.2017.10.013

31. Laory I, Trinh TN, Posenato D, Smith IFC (2013) Combined model-free data-interpretation methodologies for damage detection during continuous monitoring of structures. J Comput Civ Eng 27:657-666

32. Walker JS (1996) Fast fourier transforms, vol 24. CRC Press, Boca Raton

33. Jeong S, Ferguson M, Hou R et al (2019) Sensor data reconstruction using bidirectional recurrent neural network with application to bridge monitoring. Adv Eng Informatics 42:100991. https://doi. org/10.1016/j.aei.2019.100991

34. Posenato D, Lanata F, Inaudi D, Smith IFC (2008) Model-free data interpretation for continuous monitoring of complex structures. Adv Eng Informatics 22:135-144

35. Jolliffe IT (2002) Principal component analysis. Springer-Verlag, New York

36. Stock JH, Watson MW (1988) Testing for common trends. J Am Stat Assoc 83:1097-1107

37. Worden K, Cross E, Barton E (2012) Damage detection on the NPL Footbridge under changing environmental conditions. 6th European Workshop on Structural Health Monitoring. Dresde, Germany, pp 1-8

38. Worden K, Cross EJ, Antoniadou I, Kyprianou A (2014) A multiresolution approach to cointegration for enhanced SHM of structures under varying conditions-an exploratory study. Mech Syst Signal Process 47:243-262. https://doi.org/10.1016/j.ymssp .2013.10.012

39. Shi H, Worden K, Cross EJ (2016) A nonlinear cointegration approach with applications to structural health monitoring. J Phys Conf Ser. https://doi.org/10.1088/1742-6596/744/1/012025

40. Barton E, Middleton C, Koo K et al (2011) Structural finite element model updating using vibration tests and modal analysis for NPL Footbridge-SHM demonstrator. J Phys Conf Ser 305:012105

Publisher's Note Springer Nature remains neutral with regard to jurisdictional claims in published maps and institutional affiliations. 